\title{
Interstitials, Vacancies and Supersolid Order in Vortex Crystals
}

\author{
by \\ Erwin Frey \\ Institut für Theoretische Physik \\ Physik Department der Technischen Universität München \\ D-85747 Garching, Germany \\ David R. Nelson and Daniel S. Fisher \\ Lyman Laboratory of Physics \\ Harvard University \\ Cambridge, Massachusetts 02138
}

\begin{abstract}
Interstitials and vacancies in the Abrikosov phase of clean Type II superconductors are line imperfections, which cannot extend across macroscopic equilibrated samples at low temperatures. We argue that the entropy associated with line wandering nevertheless can cause these defects to proliferate at a sharp transition which will exist if this occurs below the temperature at which the crystal actually melts. Vortices are both entangled and crystalline in the resulting "supersolid" phase, which in a dual "boson" analog system is closely related to a two-dimensional quantum crystal of $\mathrm{He}^{4}$ with interstitials or vacancies in its ground state. The supersolid must occur for $B \gg B_{\times}$, where $B_{\times}$is the decoupling field above which vortices begin to behave two-dimensionally. Numerical calculations show that interstitials, rather than vacancies, are the preferred defect for $B \gg \phi_{0} / \lambda_{\perp}^{2}$, and allow us to estimate whether proliferation also occurs for $B \lesssim B_{\times}$. The implications of the supersolid phase for transport measurements, dislocation configurations and neutron diffraction are discussed.
\end{abstract}




\section{INTRODUCTION}

Fluctuations, especially in high-temperature superconductors, play a prominent role in determining vortex configurations in Type II materials in an external field [1]. It now appears, for example, that clean single crystal samples of YBCO (in the absence of twin boundary pinning) melt via a first-order phase transition [2] at a temperature $T_{m}$ well below the upper critical field line $\mathrm{H}_{c 2}(T)$ predicted by mean field theory [3-5]. Point disorder, in the form of oxygen vacancies, does not seem to affect this phase transition strongly in YBCO. It is quite possible that the disorder-induced translational correlation length [6] $R_{a}$ greatly exceeds the vortex spacing for $T<T_{m}$ in the field range for which the transition is first order. Although in the presence of randomness the low temperature phase is not, strictly speaking, a solid, the thermodynamic properties at and near the phase transition should be similar to those in the absence of randomness.

Consider the thermal excitations about the crystalline state on scales less than $R_{a}$. We assume for simplicity vortices which are perpendicular on average to the $\mathrm{CuO}_{2}$ planes, i.e. parallel to the $z$-direction. The finite reduction of the Debye-Waller factor associated with the translational order parameter

$$
\begin{aligned}
\rho_{\vec{G}}(T) & =\left\langle e^{i \vec{G} \cdot \vec{u}\left(\vec{r}_{\perp}, z\right)}\right\rangle \\
& =\exp \left[-\frac{1}{2} G_{i} G_{j}\left\langle u_{i}\left(\vec{r}_{\perp}, z\right) u_{j}\left(\vec{r}_{\perp}, z\right)\right\rangle\right]
\end{aligned}
$$

by phonons is discussed in Ref. 7 . Here, $\vec{G}$ is a reciprocal lattice vector and $\vec{u}\left(\vec{r}_{\perp}, z\right)$ is the displacement field of a flux lattice with vortices parallel on average to the $z$ direction. Dislocation loops are a topologically distinct excitation which, when they proliferate at a melting transition, drive $\rho_{\vec{G}}(T)$ to zero and can lead to a hexatic flux liquid with residual bond orientation order [8]. Isolated dislocation loops are far more constrained than their counterparts in crystals of point particles: dislocation loops in fact must lie in a plane spanned by their Burgers vector and the average field direction [9] - see Fig. 1a.

Vacancies and interstitials differ even more dramatically from the analogous defects in crystals of point particles. The number of flux lines is conserved, which means that these defects are lines instead of points. The point-like nature of vacancies and interstitials in conventional crystals ensures that they are present in equilibrium at all finite temperatures for entropic reasons [10]. However, because such imperfections have an energy proportional 
to their length in flux crystals, they cannot extend completely across an equilibrated macroscopic sample at low temperatures. A typical fluctuation at low temperatures might consist of the vacancy-interstitial pair shown in Fig. 1b. Unlike the dislocation loop in Fig. 1a, this loop is not constrained to lie in a single plane. This configuration also provides a mechanism by which vortices near the loop may jog one lattice constant to the right as $z$ varies.

Defect loops of this type can have important dynamical consequences. In two dimensions, point-like vacancies and interstitials probably dominate the resistive properties of a weakly pinned lattice in a superconducting film $[13,14]$. The same may well be true of vacancy-interstitial loops and lines in three dimensions as discussed in Section 5.

Although the energy of vacancy and interstitial lines is proportional to their length, it is nevertheless possible for these defects to "proliferate" (i.e., to become infinitely long) at high temperatures for entropic reasons. Consider, for example, a vacancy wandering across a macroscopic sample of thickness $L$, as in Fig. 2. To estimate the free energy of this defect, we describe its trajectory along the $z$ axis by a function $\vec{r}_{d}(z)$ and write its partition function as a functional integral,

$$
\mathcal{Z}_{d}=e^{-\epsilon_{d} L / T} \int \mathcal{D} \vec{r}_{d}(z) \exp \left\{-\frac{1}{T} \int_{0}^{L} d z\left[\frac{1}{2} \tilde{\varepsilon}_{d}\left(\frac{d \vec{r}_{d}}{d z}\right)^{2}+U_{\ell}\left(\vec{r}_{d}\right)\right]\right\}
$$

Here $\varepsilon_{d}$ is the energy of an isolated defect and $\tilde{\varepsilon}_{d}$ is its tilt energy, defined in analogy with similar quantities for isolated flux lines near $\mathrm{H}_{c 1}[7]$ and $U_{\ell}\left(\vec{r}_{d}\right)$ is a periodic lattice potential with minima at the sites of the triangular Abrikosov crystal. Implicit in the path integral (1.2) is a length scale $\ell_{z}$ which is the average distance along $z$ between hops of the vacancy from one lattice position to another. As a crude estimate of the path integral we replace it by $\exp \left(-\varepsilon_{d} L / T\right) 6^{L / \ell_{z}}$, since the vacancy has six directions in which to hop on a triangular lattice. The free energy $F_{d}=-T \ln Z_{d}$ is thus

$$
F_{d} \approx \varepsilon_{d} L-\frac{T}{\ell_{z}} L \ln 6
$$

which becomes negative for $T>T_{d}$, where

$$
T_{d}=\varepsilon_{d} \ell_{z} / \ln 6
$$

Above this temperature (provided the crystal does not melt first), vacancies (or interstitials) will proliferate in a crystalline phase. If these defects do not become strongly pinned 
by point disorder, one might then expect a linear contribution to the resistivity due to defect motion within pinned bulk crystallites, and a vortex glass transition [12] for weak pinning might then occur near $T_{d}$. More precise estimates of the proliferation temperature will be represented later in this paper. A related phenomenon was suggested by Feigel'man et al. [13], who predicted the unbinding of "quarters" (i.e., quartets) of dislocations above the decoupling field $B_{\times}$in highly anisotropic superconductors (see below).

The phase in which defects such as interstitials and vacancies proliferate is in fact both crystalline and entangled. Regarded in light of the analogy between thermally excited flux lines and two-dimensional bosons [15], it represents a "supersolid" quantum crystal, in which vacancies and interstitials are incorporated into the ground state [15-20]. The possibility of a supersolid phase for flux lines in Type II superconductors was first noted on the basis of the boson analogy by M.P.A. Fisher and Lee [21]. Somewhat paradoxically, the "supersolid" is actually less superconducting for the BCS condensate electrons than a conventional vortex lattice phase. An alternative name is an "incommensurate solid" or a "vortex density wave."

Figure 3 illustrates the connection between defect proliferation and the boson order parameter for flux lines [22]. This figure represents a low-temperature contribution to the order parameter correlation function

$$
G\left(\vec{r}_{\perp}, \vec{r}_{\perp}^{\prime} ; z, z^{\prime}\right)=\left\langle\psi\left(\vec{r}_{\perp}, z\right) \psi^{*}\left(\vec{r}_{\perp}^{\prime}, z^{\prime}\right)\right\rangle
$$

where $\psi\left(\vec{r}_{\perp}, z\right)$ and $\psi^{*}\left(\vec{r}_{\perp}^{\prime}, z^{\prime}\right)$ are destruction and creation operators for flux lines moving along the $z$ axis, which plays the role of "time" for the "bosons." The composite operator in Eq. (1.4) creates an extra line at $\left(\vec{r}_{\perp}^{\prime}, z^{\prime}\right)$ and destroys an existing line at $\left(\vec{r}_{\perp}, z\right)$. The lowest energy configuration is then a line of vacancies (for $z^{\prime}>z$, as in Fig. 3) or interstitials (for $\left.z^{\prime}<z\right)$ connecting $\left(\vec{r}_{\perp}^{\prime}, z^{\prime}\right)$ to $\left(\vec{r}_{\perp}, z\right)$. At low temperatures, the defect line joining the head to the tail costs an energy of order $f_{d}(\hat{s}) s$, where $s=\sqrt{\left(\vec{r}_{\perp}-\vec{r}_{\perp}^{\prime}\right)^{2}+\left(z-z^{\prime}\right)^{2}}$ is the length of the string in direction $\hat{s}$ and $f_{d}(\hat{s})$ is the angle-dependent defect-free energy per unit length. The correlation function (1.4) then decays exponentially to zero, like $\exp \left(-f_{d} s / T\right)$, as $\left|\vec{r}_{\perp}-\vec{r}_{\perp}^{\prime}\right| \rightarrow \infty$ with $z$ and $z^{\prime}$ fixed. Above $T_{d}, f_{d}$ changes sign, defects proliferate, and there is long-range order in $G\left(\vec{r}_{\perp}, \vec{r}_{\perp}^{\prime} ; z, z^{\prime}\right)$,

$$
\begin{aligned}
\lim _{\left|\vec{r}_{\perp}-\vec{r}_{\perp}^{\prime}\right| \rightarrow \infty} G\left(\vec{r}_{\perp}, \vec{r}_{\perp}^{\prime} ; z, z^{\prime}\right) & =\left\langle\psi\left(\vec{r}_{\perp}, z\right)\right\rangle\left\langle\psi^{*}\left(\vec{r}_{\perp}^{\prime}, z^{\prime}\right)\right\rangle \\
& \neq 0 .
\end{aligned}
$$


The physical meaning of Eq. (1.5) is that the free energy of the extra line segment from $(\vec{r}, z)$ to $\left(\vec{r}^{\prime}, z^{\prime}\right)$ in the limit of large separations remains finite. The quantity $-\ln G$ measures the free energy of a monopole anti-monopole pair at a $(\vec{r}, z)$ and $\left(\vec{r}^{\prime}, z^{\prime}\right)$ respectively. In the conventional solid phase the magnetic monopoles are confined by a linear potential, while when the defects proliferate, the monopoles are unconfined as in a normal metal with finite total free energy cost. Entanglement of vortex lines in the crystal will be catalyzed by the proliferation of vacancies and interstitials, since these allow fluxons to easily move perpendicular to the $z$ axis. Of course, entanglement will also arise if the crystal melts directly into a flux liquid. In either case the boson order parameter becomes nonzero,

$$
\psi_{0}(T)=\left\langle\psi\left(\vec{r}_{\perp}, z\right)\right\rangle \neq 0
$$

Note that Eq. (1.6) gives a precise meaning to the concept of "entanglement" as used here and elsewhere to describe "superfluid" phases within the boson analogy. Strictly speaking, the concept of "tangled vortex lines" does not distinguish sharply between low and high temperature phases for lines in an infinitely thick sample. Indeed, in all phases discussed here - solid, "supersolid" and fluid - a vortex line will wander as a function of $z$ as a random walker on sufficiently large scales. Although this wandering will be more pronounced in the higher temperature phases, it does not uniquely distinguish them from the solid. The presence or absence of particle diffusion does not sharply distinguish solids from liquids of point particles for similar reasons. Note also that labelling of vortices becomes ambiguous when the lines pass within a coherence length of each other-one must sum over "direct" and "exchange" connection possibilities to define the statistical mechanics. Entanglement also has a quantitiative meaning for dynamics: if lines can only cross or recombine by overcoming large free energy barriers, such lines are dynamically entangled, similar to a dislocation tangle in a work hardened metal. In this paper we will continue the usage in the recent literature of referring only to phases which are "superfluid" in the sense of Eq. (1.6) as entangled.

Figure 4 illustrates two distinct scenarios for phase diagrams of vortices with increasing temperature, which we call "Type I" and "Type II" melting. In Type I melting a firstorder transition separates a line crystal with $\rho_{\vec{G}} \neq 0$ from a flux liquid with $\psi_{0} \neq 0$ which may or may not be hexatic. In Type II melting, both order parameters are nonzero in an intermediate "supersolid" phase. As discussed in Sec. 4, vacancies or interstitials enter 
the Abrikosov flux lattice at $T_{d}$ in much the same way as the flux lines penetrate the Meissner phase at $\mathrm{H}_{c 1}$. Although this transition can in principle be continuous, even in the presence of strong thermal fluctuations [17], the melting of the supersolid into a liquid is likely to remain a first-order transition, since, as far as is known, continuous melting transitions in which materials lose crystallinity in two or more directions do not occur in three dimensions.

Can a real supersolid phase exist in quantum crystals? According to the review by Andreev [23], "the experimental data available at present show that the possibility ...(of a supersolid)... can hardly take place in [bulk] ${ }^{4}$ He crystals." In thin films of ${ }^{4} \mathrm{He}$, supersolid phases can, however, exist. On substrates in which one or two layers of incommensurate solid ${ }^{4} \mathrm{He}$ form, there can be a regime in which the atoms in the next partially filled layer are superfluid; this is a 2D supersolid phase even though there are almost no vacancies or interstitials in the close-packed solid layers. In 2D electron crystals at zero temperature, some kind of solid phase in which interstitials proliferate may occur [26b]. However, since electrons are fermions, such a phase would probably not be supersolid, expect perhaps at extremely low temperatures.

In the case of flux lines, the softer (longitudinal) nature of the interaction between lines makes a supersolid less unlikely than in bulk ${ }^{4}$ He. However, in the limit of continuous flux lines, where the boson analogy is best, we shall see that such a phase is still improbable. The new ingredient in the flux system, which does not have an analog for bosons, is the discreteness of the layers, i.e. discreteness in the time-like direction. As we shall see, this will definintely cause a supersolid for sufficiently large $B$ in strongly anisotropic layered superconductors. The characteristic magnetic field above which the layering becomes important is the crossover field $[12,24]$

$$
B_{\times} \sim \gamma^{2} \frac{\phi_{0}}{d_{0}^{2}},
$$

where $d_{0}$ is the layer spacing and

$$
\gamma^{2} \equiv \frac{M_{\perp}}{M_{z}}
$$

the effective mass anisotropy. Physically, this field represents the crossover between different behaviors for the energy at a "jog" where one line is shifted by an intervortex spacing $a_{0}=\left(\phi_{0} / B\right)^{1 / 2}$ from one layer to the next. A jog with shift distance $a<d_{0} / \gamma$ costs an energy quadratic in $a$, while for $a>d_{0} / \gamma$, the energy cost will be linear in $a$ and a jog 
will tend to spread out over more than one layer. For jogs with shifts $a_{0}$, the former will obtain for $B \gg B_{\times}$. In a sufficiently anisotropic system, $B_{\times} \ll H_{c 2}(T=0)$ and a regime exists, $B \gg B_{\times}$, in which the vortex fluctuations are predominantly two dimensional.

Figure 5 summarizes our conclusions about the phase diagram as function of magnetic field $B$ (i.e., vortex density) and temperature $T$, with external field $\vec{H}$ is parallel to the $\hat{c}$ axis. In a sufficiently anisotropic system, the supersolid must exist for $B \gg B_{\times}$, where vortices in different copper-oxide layers are approximately decoupled by thermal fluctuations $[12,24]$. The defects in this regime are equivalent to the "unbound dislocation quartets" discussed by Feigel'man et al. [13]. The existence of a supersolid for fields $B \lesssim B_{\times}$is a more delicate matter, which we shall discuss more quantitatively in Sec. 4 . For fields $B \gg B_{c 1} \equiv \phi_{0} / \lambda_{\perp}^{2}$, so that vortices interact via a logarithmic potential, the preferred defects are interstitials instead of vacancies. Similar conclusions were reached by Fisher et al. and by Cockayne and Elser for electrons interacting with a $1 / r$ potential in two dimensions [25]. At low fields ( $B \lesssim B_{c 1}$ ), we expect that vacancies are the favored defect, as in most crystals with short-range interactions. Although a supersolid is possible in principle for any field $B<B_{\times}$, the numerical estimates of Sec. 4 indicate that $B \gtrsim B_{\times}$ is required for this new phase to exist in high- $\mathrm{T}_{\mathrm{c}}$ superconductors.

Even if an equilibrium supersolid does not exist for $B<B_{\times}$, vacancies and interstitials may still appear for nonequilibrium reasons. If, for example, vortices pass through a firstorder freezing transition upon cooling under conditions of constant $B$, the system will initially phase separate into crystallites coexisting with a liquid of a different density. When the liquid disappears at a lower temperature, the crystal density must change to keep the macroscopic $B$ field constant. In the presence of pinning, kinetic constraints may force the crystal to absorb interstitials or vacancies rather than to changing its overall lattice constant. If freezing occurs above the field corresponding to maximum melting temperature in Fig. 5, a nonequilibrium concentration of interstitials would then result $[26]$.

In Section 2 we derive simple estimates for the defect unbinding temperature in various regimes, and compare these to the melting temperature. Numerical calculations of the energies of various kinds of straight defect lines in the high field regime are presented in Section 3. In Section 4 these energies are used to estimate $T_{d}$ more quantitatively for $B_{c 1}<$ $<B \lesssim B_{\times}$. We also discuss the consequences of supersolid order for neutron diffraction and theories of the melting transition. And, finally the implications of a supersolid phase for resistivity measurements, neutron diffraction and dislocation configurations. 


\section{ESTIMATES OF DEFECT UNBINDING TRANSITIONS}

\subsection{Model Parameters and Field Regimes}

To obtain the important physical parameters in a thermally excited vortex lattice, it is instructive to examine one representative fluxon in the confining potential provided by its surrounding vortices in a triangular lattice.

$$
\mathcal{Z}_{1}\left(\vec{r}_{\perp}, \overrightarrow{0} ; L\right)=\int_{\vec{r}(0)=\overrightarrow{0}}^{\vec{r}(L)=\vec{r}_{\perp}} \mathcal{D} \vec{r}(z) \exp \left\{-\frac{1}{T} \int_{0}^{L}\left[\frac{1}{2} \tilde{\varepsilon}_{1}\left(\frac{d \vec{r}(z)}{d z}\right)^{2}+V_{1}[\vec{r}(z)]\right] d z\right\} .
$$

As we shall see, this simple model gives predictions for melting equivalent to those obtained via "nonlocal" elastic constants and the Lindemann criterion [27]. Closely related models (such as Eq. (1.2)) will be used to determine when defects proliferate. The effective potential $V_{1}[\vec{r}(z)]$ in $(2.1)$ arises from a microscopic pairwise interaction $V\left[\vec{r}_{i j}(z)\right]$ between two parallel flux lines $\vec{r}_{i}(z)$ and $\vec{r}_{j}(z)$ with separation $\vec{r}_{i j}(z)$. In the London approximation, this potential is [1]

$$
V\left(r_{i j}\right)=2 \varepsilon_{0} K_{0}\left(r_{i j} / \lambda_{\perp}\right)
$$

where

$$
\varepsilon_{0}=\left(\phi_{0} / 4 \pi \lambda_{\perp}\right)^{2},
$$

with $\lambda_{\perp}$ the in-plane London penetration depth is the energy scale per unit length. The Bessel function $K_{0}(x) \approx \ln (x)$ for $x \ll 1$, and $K_{0}(x) \approx\left(\frac{\pi}{2 x}\right)^{1 / 2} e^{-x}$ for $x$ large. The parameter $\tilde{\varepsilon}_{1}$ is the tilt energy of a flux line, given approximately by

$$
\tilde{\varepsilon}_{1} \approx \gamma^{2} \varepsilon_{0} \ln \left(a_{0} / \xi_{\perp}\right) \quad\left(B \gg B_{c 1}\right)
$$

where $\gamma^{2} \equiv M_{\perp} / M_{z} \ll 1$ is the mass anisotropy, $a_{0}=\left(\phi_{0} / B\right)^{1 / 2}$, and $\xi_{\perp}$ is the in-plane coherence length. Equation (2.4a) follows from the wave-vector-dependent tilt modulus evaluated in the short distance regime relevant for melting [24]. This result applies only when $B \gg B_{c 1} \equiv \phi_{0} / \lambda_{\perp}^{2}$, so that we may neglect the electromagnetic coupling between $\mathrm{CuO}_{2}$ planes. When $B \lesssim B_{c 1}$, this coupling is important and the tilt energy becomes [12]

$$
\tilde{\varepsilon}_{1} \approx \varepsilon_{0}, \quad\left(B \lesssim B_{c 1}\right) .
$$

Although the tilt modulus has more generally a complicated wavevector dependence [28], it is approximately constant over the (short distance) length scales of interest to 
us here. "Nonlocal" (in $z$ ) contributions to the interaction potential [29] are similarly unimportant provided [30]

$$
\left\langle\left|\frac{d \vec{r}}{d z}\right|^{2}\right\rangle \ll \gamma^{-2},
$$

which is well satisfied throughout the crystalline phase. As discussed by Brandt [31], the same criterion justifies the neglect of higher order terms in $[d \vec{r}(z) / d z]^{2}$ in Eq. (2.1).

Three important field regimes for fluctuations in vortex crystals are easily extracted with this approach. We first rewrite this imaginary time path integral as a quantum mechanical matrix element [32],

$$
\mathcal{Z}\left(\vec{r}_{\perp}, \overrightarrow{0} ; L\right)=\left\langle\vec{r}_{\perp}\left|e^{-L \mathcal{H} / T}\right| \overrightarrow{0}\right\rangle
$$

where $|\overrightarrow{0}\rangle$ is an initial state localized at $\overrightarrow{0},\left\langle\vec{r}_{\perp}\right|$ is a final state localized at $\vec{r}_{\perp}$, and the "Hamiltonian" $\mathcal{H}$ is

$$
\mathcal{H}=-\frac{T^{2}}{2 \tilde{\varepsilon}_{1}} \nabla_{\perp}^{2}+V_{1}\left(\vec{r}_{\perp}\right) .
$$

The probability of finding the flux line at transverse position $\vec{r}_{\perp}$ within the crystal is $\psi_{0}^{2}\left(\vec{r}_{\perp}\right)$, where $\psi_{0}\left(\vec{r}_{\perp}\right)$ is the normalized ground state eigenfunction of (2.7) [33].

When $B \gg B_{c 1}$, the potential is logarithmic, and we expand $V_{1}\left(\vec{r}_{\perp}\right)$ about its minimum at $\vec{r}_{\perp}=0$ to find

$$
\left[-\frac{T^{2}}{2 \tilde{\varepsilon}_{1}} \nabla_{\perp}^{2}+\frac{1}{2} k r_{\perp}^{2}\right] \psi_{0}=\varepsilon_{0} \psi_{0}
$$

where (neglecting constants of order unity)

$$
\begin{aligned}
k & \left.\approx \frac{d^{2} V}{d r^{2}}\right|_{r=a_{0}} \\
& \approx \frac{\varepsilon_{0}}{a_{0}^{2}}
\end{aligned}
$$

and $a_{0}$ is the mean vortex spacing. Equation (2.8a) is the Schroedinger equation for a two-dimensional quantum oscillator, with $\hbar \rightarrow T$ and mass $m \rightarrow \tilde{\varepsilon}_{1}$. The ground state wave function is

$$
\psi_{0}\left(r_{\perp}\right)=\frac{1}{\sqrt{2 \pi} r_{*}} e^{-r^{2} / 4 r_{*}^{2}}
$$

with spatial extent

$$
r_{*}=\left(\frac{T^{2} a_{0}^{2}}{\varepsilon_{0} \tilde{\varepsilon}_{1}}\right)^{1 / 4} .
$$


Melting occurs when $r_{*}=c_{L} a_{0}$, where $c_{L}$ is the Lindemann constant, so the melting temperature is

$$
T_{m}=c_{L}^{2} \sqrt{\varepsilon_{0} \tilde{\varepsilon}_{1}} a_{0}, \quad\left(B_{c 1} \ll B \lesssim B_{\times}\right)
$$

in agreement with other estimates [27]. Note that $\varepsilon_{0}$ and $\tilde{\varepsilon}_{1}$ are themselves temperature dependent so that Eq. (2.11) is an implicit expression for $T_{m}(B)$, or, equivalently, a melting field $B_{m}(T)$.

This result is only applicable when $B_{m} \ll H_{c 2}\left(T_{m}\right)=\phi_{0} /\left[2 \pi \xi_{\perp}^{2}\left(T_{m}\right)\right]$ where $\xi_{\perp}(T)$ is the temperature dependent in-plane coherence length including the effects of critical thermal fluctuations which make $H_{c 2}(T) \sim\left(T_{c}-T\right)^{4 / 3}$ for $T$ sufficiently close to the zero-field transition at $T_{c}$. In this regime $\lambda_{\perp} \sim\left(T_{c}-T\right)^{-1 / 3}$ and the resulting ratio

$$
B_{m} / H_{c 2}\left(T_{m}\right)=\text { const. }
$$

independent of magnetic field or anisotropy. The logarithmic factor in $\tilde{\varepsilon}_{1}$, Eq. (2.4a), is thus of order unity since $a_{0} / \xi_{\perp}\left(T_{m}\right)=$ const. Such critical effects were not taken into account in calculations of the melting temperature prior to Ref. 12.

Vortices in the crystalline phase will travel a perpendicular distance $r_{\perp}^{*}$ in a "time" along the $z$ axis $\ell_{z}^{0}$, where [7]

$$
\begin{aligned}
\ell_{z}^{0} & \approx r_{*}^{2} /\left(T / \tilde{\varepsilon}_{1}\right) \\
& \approx \sqrt{\frac{\tilde{\varepsilon}_{1}}{\varepsilon_{0}}} a_{0} .
\end{aligned}
$$

A new high field regime arises when $\ell_{z}^{0} \lesssim d_{0}$, where $d_{0}$ is the average spacing of the copper-oxide planes, i.e., for $B \gtrsim B_{\times}$, with $[12,24]$

$$
B_{\times} \sim \frac{\tilde{\varepsilon}_{1}}{\varepsilon_{0}} \frac{\phi_{0}}{d_{0}^{2}},
$$

which is up to a logarithmic factor the same criteria discussed in the previous section, $B_{\times} \sim\left(\gamma^{2} \phi_{0} / d_{0}^{2}\right)$. Well above this field, the planes are approximately decoupled, and $T_{m}$ may be estimated from the theory of two-dimensional dislocation mediated melting $[34,12,24]$

$$
T_{m} \approx 0.5 \frac{\varepsilon_{0} d_{0}}{8 \pi \sqrt{3}}, \quad\left(B \gg B_{\times}\right)
$$


where the prefactor 0.5 is a rough estimate of the effects of phonon fluctuations [34]. Note that the convergence to Eq. (2.14) for high fields may be quite slow due to the very strongly divergent translational correlation length at the melting temperature in two dimensions $[12,24]$; this causes $T_{m}(B)$ to appraoch Eq. (2.44) only as $1 / \ln ^{2}\left(B / B_{\times}\right)$.

The estimate (2.11) also breaks down at low fields $B \lesssim B_{c 1}$ where the logarithmic interaction potential must be replaced by an exponential repulsion. The two-dimensional harmonic oscillator approximation can again be used with the replacement

$$
k \rightarrow \frac{\varepsilon_{0}}{\lambda_{\perp}^{2}} e^{-a_{0} / \lambda_{\perp}}
$$

The transverse wandering distance is now

$$
r_{*} \approx\left(\frac{T^{2} \lambda_{\perp}^{2}}{\varepsilon_{0} \tilde{\varepsilon}_{1}}\right)^{1 / 4} e^{a_{0} / 4 \lambda_{\perp}} .
$$

and takes place over a longitudinal distance

$$
\ell_{z}^{0}=\sqrt{\frac{\tilde{\varepsilon}_{1}}{\varepsilon_{0}}} \lambda_{\perp} e^{a_{0} / 2 \lambda_{\perp}} .
$$

The low field melting temperature becomes

$$
T_{m} \approx c_{L}^{2} \sqrt{\varepsilon_{0} \tilde{\varepsilon}_{1}} \frac{a_{0}^{2}}{\lambda_{\perp}} e^{-a_{0} / 2 \lambda_{\perp}} \quad\left(B \lesssim B_{c 1}\right)
$$

consistent with earlier predictions [7]. Although we have retained the distinction between $\tilde{\varepsilon}_{1}$ and $\varepsilon_{0}$ in these formulas, note from Eq. (2.4b) that $\tilde{\varepsilon}_{1} \approx \varepsilon_{0}$ in this regime.

The predictions (2.11), (2.14) and (2.18) are combined to give the reentrant phase diagram for melting shown in Fig. $5[35,12]$. We note that when fluctuations are relatively weak, so that melting occurs closer to $T_{c}$, Eqs. (2.11) and (2.18) must be solved self-consistently for $T_{m}$ using the temperature dependence of $\epsilon_{0}, \tilde{\epsilon}_{1}$ and $\lambda_{\perp}$. This procedure causes the maximum in the melting temperature to bend downwards towards $T_{c}$ in materials like YBCO [12]. $B$ rather than $H$ is used for the vertical axis since it is $B$ rather than $H$ which is fixed for the thin, low aspect ratio crystals usually studied in experiments. This figure is only schematic because a first-order transition [2] at constant $B$ would lead to two-phase coexistence with domain size limited by the strong interactions between vortex tips as they exit the sample surface in thin crystals. Analytic estimates and boundaries for melting in the various regimes are summarized in Table 1. 


\subsection{Vacancy and Interstitial Unbinding Transitions}

Consider a defect such as the vacancy shown in Fig. 2. To estimate when its free energy changes sign, we need to determine the kink separation $\ell_{z}$ which appears in Eq. (1.3b). Up to factors of order unity, a similar estimate for $T_{d}$ would apply to an interstitial vortex which, for example, hops between the centers of the triangular cells of an Abrikosov crystal. Interstitial wandering would then take place on a honeycomb lattice, and the factor $\ln 6$ in Eq. (1.4b) would be replaced by $\ln 3$.

We suppose initially that $B_{c 1} \ll B \lesssim B_{\times}$, so that the pair potential is logarithmic with well-coupled $\mathrm{CuO}_{2}$ planes. As shown in Sec. 3, the defect line energy is then proportional to the characteristic line energy scale $\varepsilon_{0}$ of the pair potential (2.2),

$$
\varepsilon_{d}=c_{d} \varepsilon_{0}
$$

In Section 3, we determine the constant $c_{d}$ for various kinds of defects, and show that in fact interstitials, rather than vacancies are favored energetically for $B \gg B_{c 1}$.

An interstitial surrounded by a triangular cage of nearest neighbors will fluctuate in much the same way as the vortex in the hexagonal cage discussed in the previous section. To "tunnel" to one of its three neighboring triangular plaquettes, it must overcome a barrier of order $\varepsilon_{0}$. The energy associated with one of these kinks or tunneling events is of order

$$
E_{k} \sim \sqrt{\varepsilon_{0} \tilde{\varepsilon}_{1}} a_{0},
$$

from Eqs. (2.12) and (2.1) since the "kinks" are spread over a distance $\ell_{z}^{0}$. This expression is just the WKB tunneling exponent which follows from the "quantum mechanical" analogy discussed above. The line-density of kinks is then of order

$$
n_{\text {kink }} \approx \frac{1}{\ell_{z}^{0}} e^{-E_{k} / T},
$$

where $\ell_{z}^{0}$ should be the same order of magnitude as in Eq. (2.12). Similar estimates would apply to vacancies: Kinks arise in Fig. 2, for example when one of the flux lines surrounding an unoccupied site jumps over a barrier in the lattice potential $U_{\ell}(\vec{r})$ of order $\varepsilon_{0}$ and essentially changes places with the vacancy. The "attempt frequency" associated with this event should again be of order $1 / \ell_{z}^{0}$. 
For both types of defects, we conclude that the spacing between kinks is of order $\ell_{z}=n_{\mathrm{kink}}^{-1}$, or

$$
\ell_{z} \sim \ell_{z}^{0} e^{E_{k} / T} .
$$

Upon substituting Eqs. (2.19) and (2.22) into (1.3b), we find a simple self-consistent equation for $T_{d}$,

$$
T_{d}=c_{1} \sqrt{\varepsilon_{0} \tilde{\varepsilon}_{1}} a_{0} \exp \left[c_{2} \sqrt{\varepsilon_{0} \tilde{\varepsilon}_{1}} a_{0} / T_{d}\right]
$$

where $c_{1}$ and $c_{2}$ are constants of order unity. It follows that

$$
T_{d}=c_{3} \sqrt{\varepsilon_{0} \tilde{\varepsilon}_{1}} a_{0}
$$

which has the same form as Eq. (2.11) but with $c_{3}$ a different numerical constant. Therefore a supersolid phase will occur whenever $c_{3}<c_{L}^{2}$. We attempt to estimate $c_{3}$ in Section 4 .

When $B \gg B_{\times}$, defects can hop one lattice constant or more between copper-oxide planes of spacing $d_{0}$, and the defect path integral (1.3) must be evaluated in a different limit. We now neglect the lattice potential $U_{\ell}\left(\vec{r}_{d}\right)$ entirely and evaluate the functional integral which remains by discretizing the path integral along $z$ in units of $d_{0}$. The result is

$$
F_{d}=\varepsilon_{d} L-\frac{T}{d_{0}} \ln \left(\frac{2 \pi B}{B_{\times}}\right) L
$$

which leads to a defect unbinding temperature

$$
T_{d}(B) \approx \text { const. } \times \varepsilon_{0} d_{0} / \ln \left[2 \pi B / B_{\times}\right] .
$$

Since $T_{d} \sim 1 / \ln \left(2 \pi B / B_{\times}\right)$in this regime, while $T_{m}$ is asymptotically $B$-independent according to Eq. (2.14), defects must proliferate for $B \gg B_{\times}$. Equation (2.26) agrees with the unbinding transition of dislocation pairs from bound dislocation quartets estimated in Ref. 13 .

The result Eq. (2.26) for the limit $B_{\times} \gg B$ can also be obtained from a simple physical argument. In the limit of nearly decoupled layers with a very weak Josephson coupling, the density of defects in each layer is just $n_{d} \sim e^{-E_{d} / T}$ where

$$
E_{d}=d_{0} \varepsilon_{d},
$$

is the energy of the defect in a single layer. When the interactions between layers are weak, they can be estimated perturbatively. If a vortex passing through two neighboring layers 
is misaligned by an amount $b$, the excess cost is of order $\gamma^{2} \varepsilon_{0} b / d_{0}$ from the Josephson coupling between the layers. Naively, the cost of misalignment of the vortex which makes up an interstitial "line" is obtained by setting $b=a_{0} n_{d}^{-1 / 2}$, the typical jog distance of the defect between nearly decoupled layers. This is certainly an upper bound for the kink-free energy since fluctuations of the other vortices in each layer will add a large incoherent part to the phase difference associated with the interstitial. We conjecture that this will result in a reduction of the full Josephson energy to

$$
E_{I} \sim \frac{\gamma^{2} \varepsilon_{0} a_{0}^{2} n_{d}^{-k}}{d_{0}}
$$

with $k \leq 1$. The free energy per layer of the defects is then

$$
F_{d} \approx E_{d} n_{d}+T n_{d} \ln n_{d}+n_{d} E_{I}\left(n_{d}\right) .
$$

Minimizing with respect to $n_{d}$ yields the result that $F_{d}$ becomes negative, and hence $n_{d}$ positive, when $T>T_{d}$ with

$$
T_{d} \approx k E_{d} / \ln \left(B / B_{\times}\right)
$$

just the form of Eq. (2.26). Note that the magnetic interlayer coupling will only add an $n_{d}$ independent term to $E_{I}$ because of the screening due to relaxation at the other vortices as discussed in Appendix E.

The $2 \mathrm{D}$ defect energy $E_{d}$ is correctly obtained via Eq. (2.27) from the $3 D$ calculations of $E_{d}$ described in this paper since in the absence at Josephson coupling the vortices in 2D layers interact logarithmically at all distances with the effective penetration length at long distances only reduced by negligible $\mathcal{O}\left(d_{0} / \lambda_{\perp}\right)$ corrections for small $d_{0}$.

Finally, when $B \lesssim B_{c 1}$, the exponential interaction between vortices leads to a different result. The lowest energy defect in this regime of short-range interactions is presumably a vacancy, as is usual for solids with short-range interactions. The characteristic defect line energy is now

$$
\varepsilon_{d}=\text { const. } \times \frac{a_{0}^{2}}{\lambda_{\perp}^{2}} \varepsilon_{0} e^{-a_{0} / \lambda_{\perp}} .
$$

The barrier to produce a kink in the trajectory of a vacancy or interstitial has similar exponential dependence on $a_{0}$, so the resulting kink energy is

$$
E_{k} \sim \sqrt{\varepsilon_{0} \tilde{\varepsilon}_{1}} \frac{a_{0}^{2}}{\lambda_{\perp}} e^{-a_{0} / 2 \lambda_{\perp}}
$$


Upon using Eq. (2.17) for $\ell_{z}^{0}$ in Eq. (2.22), we find via Eq. (1.30) that

$$
F_{d}=\varepsilon_{d} L\left[1-\text { const. } \times \frac{T}{E_{k}} e^{-E_{k} / T}\right]
$$

which (provided the constant is not too small) changes sign for

$$
\begin{aligned}
T_{d} & \approx \text { const. } E_{k} \\
& \approx \text { const. } \times \sqrt{\varepsilon_{0} \tilde{\varepsilon}_{1}}\left(\frac{a_{0}^{2}}{\lambda_{\perp}}\right) e^{-a_{0} / 2 \lambda_{\perp}}
\end{aligned}
$$

in the limit of low fields. A supersolid phase is in principle possible for low fields if $T_{d}<T_{m}$ as given by Eq. (2.18). We conclude in Sec. 4 however, that $T_{m}<T_{d}$ for $B \lesssim B_{\times}$, which makes this possibility rather unlikely.

Our estimates for interstitial or vacancy proliferation temperatures are summarized in Table 1. Combining these estimates for $T_{d}$ and $T_{m}$ (and assuming that the supersolid is unfavorable for small fields) leads to the schematic phase diagram shown in Fig. 5. 


\section{FORMATION ENERGY OF LINE DEFECTS}

In this section we discuss more precisely the defect line energy $\epsilon_{d}$ for rigid configurations of straight vortex lines. Numerical calculations are presented for various types of defects in the limit $B_{c 1} \ll B \ll H_{c 2}$.

\subsection{Gibbs Free Energy of a Perfect Vortex Lattice}

Let $\vec{x}(\ell)$ be the equilibrium position of the $\ell$ th flux line. In the London limit, $\kappa=$ $\lambda_{\perp} / \xi_{\perp} \gg 1$, the pair potential between two straight flux lines is given by Eq. (2.2),

$$
V\left(r_{\ell \ell^{\prime}}\right)=\frac{\phi_{0}^{2}}{8 \pi^{2} \lambda_{\perp}^{2}} K_{0}\left(r_{\ell \ell^{\prime}} / \lambda_{\perp}\right)
$$

where $r_{\ell \ell^{\prime}}=\left|\vec{x}(\ell)-\vec{x}\left(\ell^{\prime}\right)\right|$, and $K_{0}$ is the modified Bessel function, with the asymptotic behavior $K_{0}(x) \approx(\pi / 2 x)^{1 / 2} e^{-x}$ for large $\mathrm{x}$ and $K_{0}(x) \approx-\ln (x / 2)-\gamma$ for small $x$.

For $\vec{H}\|\vec{B}\| \vec{c}$ the Gibbs free energy per unit length $L$ and unit area $A$ is

$$
g=n\left(\epsilon_{1}-\frac{H \phi_{0}}{4 \pi}\right)+n \epsilon_{0} \sum_{\ell}^{\prime} K_{0}\left(r_{0 \ell} / \lambda_{\perp}\right)
$$

Here $\epsilon_{1}=\left(\frac{\phi_{0}}{4 \pi \lambda_{\perp}}\right)^{2} \ln \kappa=\epsilon_{0} \ln \kappa$ is the energy per unit length of a single flux line (ignoring an additive constant correction to $\ln \kappa)$, and $n=N / A$ is the number of lines per unit area. The second term in the Gibbs free energy represents the interaction energies of the lines, while the first includes the effect of the external magnetic field $H$ and favors large values of $B . H$ plays the role of a pressure (or chemical potential), which tends to increase the density of lines. The prime denotes that self-interactions (the $\ell=0$ term) are omitted from the summation. The equilibrium magnetic flux density $B_{\mathrm{eq}}=n \phi_{0}$ is obtained by minimizing the Gibbs free energy, i.e., by solving $d G / d B=0$. In the limit $B_{\text {eq }} \gg \phi_{0} / \lambda_{\perp}^{2}$ one finds [36]

$$
H-H_{c 1}=B_{\mathrm{eq}}+\frac{\phi_{0}}{8 \pi \lambda_{\perp}^{2}}\left[\ln \left(\frac{\phi_{0}}{B_{\mathrm{eq}} \lambda_{\perp}^{2}}\right)+C\right]+\mathcal{O}\left(B_{\mathrm{eq}}^{-1}\right)
$$

where $H_{c 1}=\frac{4 \pi \epsilon_{1}}{\phi_{0}}$. The constant $C=-\ln 4 \pi-2+\gamma+A$ depends on the lattice structure with $\gamma \approx 0.5772 .$. is Euler's constant. For a hexagonal and square lattice we find $A_{6} \approx$ 
0.0797107, and $A_{4} \approx 0.1008797$, respectively [36]. For a rectangular lattice with lattice constants $L_{x}$ and $L_{y}$ the value of $A$ has to be calculated from

$$
A=\sum_{\vec{R}}^{\prime} E_{1}\left(\frac{\pi \vec{R}^{2}}{L_{x} L_{y}}\right)+\sum_{\vec{G}}^{\prime} \frac{\exp \left[-\vec{G}^{2} L_{x} L_{y} / 4 \pi\right]}{\vec{G}^{2} L_{x} L_{y} / 4 \pi} .
$$

The corresponding minimum value of the Gibbs free energy is

$$
G_{\mathrm{eq}}=-\frac{B_{\mathrm{eq}}^{2}}{8 \pi}+\frac{B_{\mathrm{eq}} \phi_{0}}{32 \pi^{2} \lambda_{\perp}^{2}}+\mathcal{O}(1)
$$

We are interested in the formation energy of "point defects" at constant line density $n$ corresponding to a fixed external magnetic field $H$. This later condition allows us to consider only the interaction part of the free energy (3.2) for $N$ particles, i.e.,

$$
E_{N}=N \epsilon_{0} \sum_{\ell}^{\prime} K_{0}\left(r_{0 \ell} / \lambda_{\perp}\right)
$$

in determining defect energies. With Eqs. (3.2)-(3.5) one finds

$$
E_{N}=2 N \epsilon_{0}\left\{n \pi \lambda_{\perp}^{2}+\frac{1}{4}\left[\ln \left(\frac{1}{n \lambda_{\perp}^{2}}\right)+C+1\right]\right\}
$$

\subsection{Definition of Defect Energies}

\subsubsection{Constant lattice spacing}

We follow Ref. 25 and illustrate the definition of defect energies for the case of a vacancy line. Consider first a perfect lattice with $N+1$ flux lines confined to an area $A$. The corresponding interaction energy is $E_{N+1}$. Removing a line at the origin without allowing the flux lattice to relax reduces the interaction energy to

$$
E^{\prime}=\frac{N+1}{2} \sum_{\ell}^{\prime} V\left(r_{0 \ell}\right)-\sum_{\ell}^{\prime} V\left(r_{0 \ell}\right)
$$

i.e., we have subtracted the interaction energy of the removed center line with the rest of the lines. Hence the (unrelaxed) defect energy for a vacancy at constant lattice spacing is given by

$$
E_{\mathrm{V}}^{(2)}=E^{\prime}-E_{N+1}=-\frac{2}{N+1} E_{N+1}
$$




\subsubsection{Constant line density}

The vacancies that occur in a superconductor occur at constant line density or constant chemical potential, not constant lattice constant. Starting with a large perfect lattice containing $N$ flux lines in a fixed area $A$ one can imagine rearranging the flux lines (with $N$ fixed) in such a way that the resulting configuration is identical to the one generated above by removing one line from a perfect lattice containing $N+1$ lines. Thus, the unrelaxed vacancy energy at constant line density is defined by [25]

$$
E_{\mathrm{V}}^{u}=E^{\prime}-E_{N}=E_{\mathrm{V}}^{(2)}+\left(E_{N+1}-E_{N}\right) .
$$

Upon using Eqs. (3.2)-(3.7) one gets for the Gibbs free energy per unit length of a vacancy line in an unrelaxed lattice,

$$
G_{\mathrm{V}}^{u}=E_{\mathrm{V}}^{u}-\frac{\epsilon_{0}}{2}\left[\ln \left(\frac{1}{n \lambda_{\perp}^{2}}\right)+C+2\right] .
$$

Note that we have neglected terms of the order $n / N=1 / A$ since we are interested in the thermodynamic limit with $n$ fixed and $N \rightarrow \infty$. The defect energy itself is $\epsilon_{\mathrm{V}}=G_{\mathrm{V}} \sim$

$\epsilon_{0}\left[\ln \left(\frac{\lambda_{\perp}}{a_{0}}\right)+\right.$ const. $]$ which is the correct order of magnitude except for the logarithmic divergence as $a_{0} / \lambda_{\perp} \rightarrow 0$. As we shall see, this singularity disappears when we allow the lattice to relax around the defect.

\subsection{Elasticity Theory}

Before allowing static relaxations, we review the energies of the phonon distortions which are involved. When each vortex line is given an arbitrary displacement $\vec{u}(\ell)$ from its equilibrium position $\vec{x}(\ell)$ the interaction (potential) energy of the vortex crystal is given by

$$
E=\frac{1}{2 A} \sum_{\ell, \ell^{\prime}}^{\prime} V\left(\left|\vec{x}\left(\ell \ell^{\prime}\right)+\vec{u}\left(\ell \ell^{\prime}\right)\right|\right)
$$

where $\vec{x}\left(\ell \ell^{\prime}\right)=\vec{x}(\ell)-\vec{x}\left(\ell^{\prime}\right)$ and $\vec{u}\left(\ell \ell^{\prime}\right)=\vec{u}(\ell)-\vec{u}\left(\ell^{\prime}\right)$ are the differences in the lattice vectors and displacement vectors, respectively. Expanding up to second order in the displacement fields yields

$$
E=E_{N}+\frac{1}{2 A} \sum_{\ell, \alpha} \sum_{\ell^{\prime}, \beta} \phi^{\alpha \beta}\left(\ell, \ell^{\prime}\right) u^{\alpha}(\ell) u^{\beta}\left(\ell^{\prime}\right)+\ldots
$$


where $E_{N}$ is the interaction energy of a rigid flux lattice. Here $\alpha, \beta=x, y$ label the Cartesian components and the dynamic matrix is given by

$$
\phi^{\alpha \beta}\left(\ell, \ell^{\prime}\right)= \begin{cases}-\frac{\partial^{2} V\left(r_{\ell \ell^{\prime}}\right)}{\partial x^{\alpha} x^{\beta}} & \text { for } \ell \neq \ell^{\prime} \\ \sum_{\ell \neq \ell^{\prime}} \frac{\partial^{2} V\left(r_{\ell \ell^{\prime}}\right)}{\partial x^{\alpha} x^{\beta}} & \text { for } \ell=\ell^{\prime} .\end{cases}
$$

Upon defining the Fourier transform

$$
C^{\alpha \beta}(\vec{q})=a_{c} \sum_{\ell} \phi^{\alpha \beta}(\ell, 0) e^{-i \vec{q} \cdot \vec{x}(\ell, 0)}=-\left(S^{\alpha \beta}(\vec{q})-S^{\alpha \beta}(0)\right)
$$

with

$$
\begin{aligned}
S^{\alpha \beta}(\vec{q}) & =a_{c} \sum_{\ell\left(\neq \ell^{\prime}\right)} \frac{\partial^{2} V\left(r_{\ell \ell^{\prime}}\right)}{\partial x^{\alpha} \partial x^{\beta}} e^{-i \vec{q} \cdot \vec{x}\left(\ell, \ell^{\prime}\right)} \\
& =a_{c} \lim _{\vec{x} \rightarrow 0} \frac{\partial^{2}}{\partial x^{\alpha} \partial x^{\beta}}\left[e^{-i \vec{q} \cdot \vec{x}} \sum_{\ell} e^{-i \vec{q} \cdot(\vec{x}(\ell)-\vec{x})} V(\vec{x}(\ell)-\vec{x})-V(\vec{x})\right]
\end{aligned}
$$

the interaction energy can be written as

$$
E=E_{N}+\frac{1}{2 A a_{c}^{2}} \int_{\vec{q}} u^{\alpha}(\vec{q}) C^{\alpha \beta}(-\vec{q}) u^{\beta}(-\vec{q}) .
$$

Here $a_{c}=\frac{\sqrt{3}}{2} a_{0}^{2}=n^{-1}$ is the volume of the unit cell of the triangular lattice and we have introduced the short hand notation $\int_{\vec{q}}=\int \frac{d^{2} q}{(2 \pi)^{2}}$ for integrals over the in-plane wave vector $\vec{q}$.

In the limit of a very large London penetration depth, $\lambda_{\perp} \gg a_{0}$, the dynamic matrix takes the form (see Appendix A)

$$
C^{\alpha \beta}(\vec{q})=2 \epsilon_{0}\left\{2 \pi \frac{q^{\alpha} q^{\beta}}{q^{2}}+\frac{a_{c}}{8}\left[\delta^{\alpha \beta} q^{2}-2 q^{\alpha} q^{\beta}\right]\right\}
$$

for small values of $q$. Upon comparing with the usual continuum elastic description of vortex solids [27], $C^{\alpha \beta}(\vec{q})=a_{c}^{2}\left[c_{1} 1(\vec{q}) q^{\alpha} q^{\beta}+c_{66}(\vec{q}) \delta^{\alpha \beta} q^{2}\right]$, we find that the bulk and shear moduli are

$$
\begin{gathered}
c_{11}(\vec{q}) \approx n^{2} \frac{4 \pi \epsilon_{0}}{q^{2}}-\frac{n \epsilon_{0}}{2} \\
c_{66}(\vec{q}) \approx \frac{n \epsilon_{0}}{4} .
\end{gathered}
$$


where $n=a_{c}^{-1}$ is the vortex density. In this limit of rigid, parallel vortices we obtain no information on the $q_{z}$-dependence of these quantities or on the tilt modulus $c_{44}$. Equation (3.20) agrees with the shear modulus first obtained by Fetter et al. [38]. Equation (3.19) is correct for $\lambda_{\perp}^{-1}<q_{\perp}<a_{0}^{-1}$, which encompasses a wide range of length scales when $B \gg \phi_{0} / \lambda_{\perp}^{2}$.

\subsection{Variational Calculation of the Relaxational Energy of a Straight Vacancy Line}

In this section we calculate the relaxation energy of a straight vacancy line by a variational approach. The presence of a vacancy causes a distortion of the lattice described by the displacement field $\vec{u}(\ell)$. Assuming the displacements $\vec{u}(\ell)$ to be small and slowly varying the relaxational energy for a vacancy can be obtained by minimization with respect to $\vec{u}(\ell)$ of

$$
\begin{aligned}
E_{\mathrm{VR}}^{(2)}(\{\vec{u}\})= & \frac{1}{2} \sum_{\ell, \ell^{\prime}} \phi^{\alpha \beta}\left(\ell, \ell^{\prime}\right) u^{\alpha}(\ell) u^{\beta}\left(\ell^{\prime}\right) \\
& -1 \sum_{\ell \neq 0} V_{1}^{\alpha}(\ell) u^{\alpha}(\ell) \\
& -\frac{1}{2} \sum_{\ell \neq 0} V_{2}^{\alpha \beta}(\ell) u^{\alpha}(\ell) u^{\beta}(\ell),
\end{aligned}
$$

where

$$
\begin{aligned}
V_{1}^{\alpha}(\ell) & =\frac{\partial}{\partial x^{\alpha}} V(\vec{x}) \\
V_{2}^{\alpha \beta}(\ell) & =\frac{\partial^{2}}{\partial x^{\alpha} \partial x^{\beta}} V(\vec{x})
\end{aligned}
$$

and the dynamical matrix $\phi^{\alpha \beta}\left(\ell, \ell^{\prime}\right)$ is defined in Eq. (3.14).

The first term in $E_{\mathrm{VR}}(\{\vec{u}\})$ is the elastic energy of the lattice distortion caused by the vacancy. It is overcounts the energy of the missing center line with the rest of the crystal. This contribution is subtracted by the second and third term in Eq. (3.21), which is just a Taylor expansion of $\sum_{\ell \neq 0} V(|\vec{x}(\ell)+\vec{u}(\ell)-\vec{x}(0)|)$, where $\vec{x}(0)=0$ is the position of the vacancy in the lattice.

In Fourier space, we have

$$
u^{\alpha}(\vec{q})=a_{c} \sum_{\ell} u^{\alpha}(\ell) e^{-i \vec{q} \cdot \vec{x}(\ell)}
$$




$$
\begin{aligned}
C^{\alpha \beta}(\vec{q}) & =a_{c} \sum_{\ell} \phi_{\alpha \beta}(\ell, 0) e^{-i \vec{q} \cdot \vec{x}(\ell)} \\
V_{1}^{\alpha}(\vec{q}) & =a_{c} \sum_{\ell \neq 0} V_{1}^{\alpha}(\ell) e^{-i \vec{q} \cdot \vec{x}(\ell)} \\
V_{2}^{\alpha \beta}(\vec{q}) & =a_{c} \sum_{\ell \neq 0} V_{2}^{\alpha \beta}(\ell) e^{-i \vec{q} \cdot \vec{x}(\ell)}
\end{aligned}
$$

and the vacancy relaxation energy becomes

$$
\begin{aligned}
E_{\mathrm{VR}}^{(2)}(\{\vec{u}(\ell)\}) & =\frac{1}{2 a_{c}^{2}} \int_{q} u^{\alpha}(\vec{q}) u^{\beta}(-\vec{q}) C^{\alpha \beta}(-\vec{q}) \\
& -\frac{1}{a_{c}} \int_{q} V_{1}^{\alpha}(\vec{q}) u^{\alpha}(-\vec{q}) \\
& -\frac{1}{2 a_{c}} \int_{q} \int_{k} V_{2}^{\alpha \beta}(\vec{q}-\vec{k}) u^{\alpha}(\vec{k}) u^{\beta}(-\vec{q}) .
\end{aligned}
$$

Following Ref. 25, we make a variational Ansatz for the lattice distortion field,

$$
u^{\alpha}(\vec{q})=i a_{c} \frac{q^{\alpha}}{q^{2}} f(q)
$$

where $f(q)=1+c q+d q^{2}$ with $c$ and $d$ as variational parameters. As discussed in Appendix B, the constraint $f(0)=1$ is enforced by the long range potential. $V_{1}^{\alpha}(\vec{q})$ and $V_{2}^{\alpha \beta}(\vec{q})$ can be expressed in terms of the dynamic matrix $C^{\alpha \beta}(\vec{q})$

$$
\begin{gathered}
V_{1}^{\alpha}(\vec{q})=a_{c} \sum_{\ell \neq 0} V_{1}^{\alpha}(\ell) e^{-i \vec{q} \cdot \vec{x}(\ell)}=-i \frac{\partial}{\partial q_{\gamma}} V_{2}^{\alpha \gamma}(\vec{q}), \\
V_{2}^{\alpha \beta}(\vec{q})=a_{c} \sum_{\ell \neq 0} V_{2}^{\alpha \beta}(\ell) e^{-i \vec{q} \cdot \vec{x}(\ell)}=-C^{\alpha \beta}(\vec{q})+V_{2}^{\alpha \beta}(\vec{q}=0),
\end{gathered}
$$

where we have used the asymptotic form of the potential, $\lim _{\lambda_{\perp} \rightarrow \infty} V(x)=-\ln x$, to derive equation (3.30). This gives

$$
V_{1}^{\alpha}(\vec{q})=2 i \varepsilon_{0}\left(\frac{2 \pi}{q^{2}}-\frac{a_{c}}{2}\right) q^{\alpha},
$$

and for the above Ansatz for the displacement field one can take

$$
V_{2}^{\alpha \beta}(\vec{q})=-C^{\alpha \beta}(\vec{q})
$$

Upon using the long wavelength approximation $(2.18)$ for $C^{\alpha \beta}(\vec{q})$, one finds

$$
E_{\mathrm{VR}}^{(2)}(\{\vec{u}(\ell)\})=2 \varepsilon_{0}\left(I_{1}+I_{2}+I_{3}\right)
$$


with $I_{i}$ given in Appendix B. The variational calculation for $c$ and $d$ yields (see Appendix B)

$$
\begin{gathered}
c=\frac{1}{7 k_{d}} \approx 0.161 \sqrt{a_{c}}, \\
d=-\frac{10}{7 k_{d}^{2}} \approx-0.114 a_{c},
\end{gathered}
$$

where $k_{d}=\sqrt{4 \pi / a_{c}}$ is a cutoff which preserves the area of the underlying hexagonal Brillouin zone. Hence, the elastic relaxation contribution to the vacancy energy density at constant lattice spacing is

$$
E_{\mathrm{VR}}^{(2)}=\frac{\varepsilon_{0}}{2}\left[\ln \left(\frac{1}{k_{d}^{2} \lambda_{\perp}^{2}}\right)+\frac{265}{252}\right]
$$

The total vacancy energy density at constant density instead of constant lattice constant, is, following the considerations of Sec. 3.2,

$$
G_{V}=E_{\mathrm{VR}}^{(2)}+E_{V}^{(2)}+E_{N+1}-E_{N} .
$$

The last three terms are given by Eq. (3.11), which, when combined with (3.37), leads to

$$
G_{V}=\frac{\varepsilon_{0}}{2}\left[-\gamma-A_{6}+\frac{265}{252}\right] .
$$

Our variational vacancy line-energy is thus $\epsilon_{V}=G_{V}$, or

$$
\epsilon_{V}=0.1973 \varepsilon_{0}
$$

Note that the logarithmic divergences as $a_{0} / \lambda_{\perp} \rightarrow 0$ in Eqs. (3.11) and (3.37) have cancelled to yield a finite result.

A heuristic "back of the envelope" argument for the vacancy energy can also be constructed: assume that the phonon displacement in real space for a vacancy at the origin takes the isotropic form

$$
\vec{u}(\ell)=-\frac{\Omega_{0}}{2 \pi} \frac{\vec{x}(\ell)}{x^{2}(\ell)},
$$

consistent with a six-fold symmetry of this defect site [37]. The parameter $\Omega_{0}$ is the area change induced in the flux line lattice. To keep the density of flux lines fixed (as is appropriate for $B \gg \phi_{0} / \lambda_{\perp}^{2}$ ), we take $\Omega_{0}=a_{c}$, the area of one unit cell to cancel the vacancy energy. Since $\boldsymbol{\nabla} \cdot \vec{u}=0$ and $\partial_{z} \vec{u}=0$, the only contribution from the continuum 
elastic free energy per unit length comes from the wave-vector independent shear modulus term,

$$
\epsilon_{V}=\int d^{2} x c_{66} u_{i j}^{2}
$$

where $u_{i j}=\frac{1}{2}\left(\partial_{i} u_{j}+\partial_{j} u_{i}\right), i=1,2$ and [38] $c_{66}=\frac{1}{4} n_{0} \varepsilon_{0}$. In Fourier space, this expression becomes

$$
\epsilon_{V}=c_{66} \int \frac{d^{2} q}{(2 \pi)^{2}}\left|u_{i j}(\vec{q})\right|^{2}
$$

with $u_{i j}(\vec{q})=-a_{c} q_{i} q_{j} / q^{2}$. Upon using Eq. (3.20) and imposing a circular Brillouin zone of radius $k_{d}=\sqrt{4 \pi / a_{c}}$, we find finally

$$
\epsilon_{V}=\varepsilon_{0} / 4
$$

an estimate only $25 \%$ greater than Eq. (3.40), and very close to the numerical value for the relaxed vacancy configuration with six-fold symmetry [37].

\subsection{Numerical Calculation of the Defect Energies}

Interstitial defects generally occupy lattice sites of low symmetry, so that analytic methods become quite laborious. In this section we describe numerical calculations of the various defect energies which do not require the approximations used above. Our goal is to calculate the defect energies for an infinite system.

In the computer simulations, we have not an infinite system but a system with a large but finite block of particles with periodic boundary conditions. In order to handle the longrange logarithmic interaction between the flux lines we use the the Ewald sum technique, which amounts to including the interaction of the flux line with all its periodic images [39]. For comparison with the computer results, one must therefore calculate not the energy of a single defect in an infinite system, but the energy per block of an periodic array of defects in an infinite system. After correcting for these "image" defects one can extract the desired energy of a single defect. In practice we choose the system large enough that the interaction of the defects with its periodic images can either be neglected or calculated by means of linear elastic theory.

The interaction energy of the flux line lattice per unit length in the $z$ direction is

$$
E=\frac{E_{0}}{2} \sum_{\ell, \ell^{\prime}} K_{0}\left(r_{\ell \ell^{\prime}} / \lambda_{\perp}\right)
$$


where our energy unit is $E_{0}=2 \varepsilon_{0}$. This sum over the infinite lattice can also be written as

$$
E=\frac{E_{0}}{2} \sum_{\ell, \ell^{\prime} \in \text { box }} \phi\left(r_{\ell \ell^{\prime}} / \lambda_{\perp}\right)
$$

where the summation is over all particles within the basic box of size $\left(L_{x}, L_{y}\right)$. The effective pair potential within the basic box

$$
\phi\left(r_{\ell \ell^{\prime}} / \lambda_{\perp}\right)=\sum_{\vec{R}}^{\prime} K_{0}\left(\left|\vec{x}\left(\ell \ell^{\prime}\right)+\vec{R}\right| / \lambda_{\perp}\right)
$$

represents the interaction energy between a flux line at position $\vec{x}(\ell)$ and one at position $\vec{x}\left(\ell^{\prime}\right)$ together with all its images at positons $\vec{x}\left(\ell^{\prime}\right)+\vec{R}$. The sum over $\vec{R}$ runs over all simple cubic lattice points, $\vec{R}=\left(\ell L_{x}, m L_{y}\right)$ with $\ell, m$ integers. This vector reflects the shape of the basic box. The prime on the summation sign of Eq. (3.47) indicates that we omit the term $\vec{R}=0$ for $\vec{x}\left(\ell \ell^{\prime}\right)=0$.

For a numerical calculation equation (3.47) is not a suitable starting point because of its poor convergence properties. Therefore, we make use of Ewald's summation method [39]. Using the integral representation of the modified Bessel function $K_{0}$ and Ewald's generalized theta function transform (see Appendix A) we find for $\vec{x} \neq 0$ in the limit of large $\lambda$

$$
\begin{aligned}
\phi(\vec{x} / \lambda)=\frac{1}{2} \sum_{\vec{R}} E_{1}\left(\frac{(\vec{x}-\vec{R})^{2} \pi}{L_{x} L_{y} \delta}\right) \\
+\frac{1}{2} \sum_{\vec{G}}^{\prime} e^{-i \vec{G} \cdot \vec{x}} \frac{\exp \left[-L_{x} L_{y} G^{2} \delta / 4 \pi\right]}{L_{x} L_{y} G^{2} / 4 \pi} \\
+\frac{2 \pi \lambda^{2}}{L_{x} L_{y}}-\frac{\delta}{2},
\end{aligned}
$$

where $E_{1}(x)=\int_{0}^{\infty} d y y^{-1} e^{-y}$ is the exponential integral function. The vectors $\vec{G}=$ $2 \pi\left(m / L_{x}, n / L_{y}\right)$ with $m$ and $n$ integers index the square lattice reciprocal to the lattice of image lines. Note that this result is valid for any choice of the Ewald separation parameter $\delta$. It can be used as a numerical check and to optimize the convergence properties of Eq. (3.48). We choose $\delta=1$. 
For $\phi(0)$ we get

$$
\begin{aligned}
\phi(0) & =\frac{1}{2} \sum_{\vec{R}}^{\prime} E_{1}\left(\frac{\pi R^{2}}{L_{x} L_{y}}\right)+\frac{1}{2} \sum_{\vec{G}}^{\prime} \frac{\exp \left[-G^{2} L_{x} L_{y} / 4 \pi\right]}{G^{2} L_{x} L_{y} / 4 \pi}+\frac{2 \pi \lambda_{\perp}^{2}}{L_{x} L_{y}}-\frac{1}{2}+\frac{1}{2} \ln \frac{L_{x} L_{y}}{4 \pi \lambda_{\perp}^{2}}+\frac{\gamma}{2} \\
& =\frac{2 \pi \lambda_{\perp}^{2}}{L_{x} L_{y}}+\frac{1}{2} \ln \left(\frac{L_{x} L_{y}}{\lambda_{\perp}^{2}}\right)+\frac{1}{2}\left(\gamma-1-\ln 4 \pi+A_{\text {rect }}\right) .
\end{aligned}
$$

The value of the Ewald sum $A_{\text {rect }}$ depends on the shape of the basic box (compare with Eq. (3.4)). We choose the rectangular basic box such that $L_{x}=5 a_{0} \cdot t$ and $L_{y}=3 \sqrt{3} a_{0} \cdot t$ with $t$ integer. Then one gets from a numerical evaluation of the Ewald sums $A_{\text {rect }} \approx$ 0.1018412 and the interaction energy in units of $E_{0}$ becomes

$$
E=\frac{N^{2}}{2} \frac{2 \pi \lambda_{\perp}^{2}}{L_{x} L_{y}}+\frac{N}{4}\left[\gamma-1-\ln 4 \pi+A_{\text {rect }}+\ln \left(\frac{L_{x} L_{y}}{\lambda_{\perp}^{2}}\right)\right]+\frac{1}{2} \sum_{\ell, \ell^{\prime}}^{\prime} \hat{\Phi}\left(r_{\ell \ell^{\prime}} / \lambda_{\perp}\right)
$$

with the effective pair potential

$$
\hat{\Phi}\left(r_{\ell \ell^{\prime}}\right)=\frac{1}{2} \sum_{\vec{R}} E_{1}\left(\frac{(\vec{x}-\vec{R})^{2} \pi}{L_{x} L_{y}}\right)+\frac{1}{2} \sum_{\vec{G}}^{\prime} e^{-i \vec{G} \cdot \vec{x}} \frac{\exp \left[-L_{x} L_{y} G^{2} / 4 \pi\right]}{L_{x} L_{y} G^{2} / 4 \pi}-\frac{1}{2} .
$$

Upon comparing Eq. (3.50) with the interaction energy of a perfect flux line lattice, Eq. (3.7), one gets for a rectangular basic box

$$
\frac{1}{2} \sum_{\ell, \ell^{\prime}}^{\prime} \hat{\Phi}\left(r_{\ell \ell^{\prime}}\right)=\frac{N}{4}\left(-\ln N+A_{6}-A_{\text {rect }}\right)
$$

where $N=30 \cdot t^{2}$ is the number of flux lines in the box of size $\left(L_{x}, L_{y}\right)=(5,3 \sqrt{3}) t a_{0}$.

For the numerical calculations it is sufficient to consider only those parts of the interaction energy, which explicitely depend on the coordinates of the flux lines. Hence we consider the quantity

$$
\hat{E}=\frac{1}{2} \sum_{\ell, \ell^{\prime}} \hat{\Phi}\left(r_{\ell \ell^{\prime}}\right)
$$

The force between flux lines at a distance $\vec{x}\left(\ell \ell^{\prime}\right)$ is given by

$$
\vec{F}\left(\vec{x}_{\ell \ell^{\prime}}\right)=\vec{F}_{\ell \ell^{\prime}}=-\frac{\partial}{\partial \vec{x}\left(\ell \ell^{\prime}\right)} \hat{\Phi}\left(r_{\ell \ell^{\prime}}\right)
$$

Using the above expression for the interaction potential $\hat{\Phi}$ one can write

$$
\begin{aligned}
\vec{F}(\vec{x})=\frac{1}{2}\left\{\sum_{\vec{R}} \frac{2 \pi}{L^{2}} \frac{\exp \left[-|\vec{x}-\vec{R}|^{2} \pi / L_{x} L_{y}\right]}{|\vec{x}-\vec{R}|^{2} \pi / L_{x} L_{y}}(\vec{x}-\vec{R})\right. \\
\left.+\sum_{\vec{G}}^{\prime} \vec{G} \sin (\vec{G} \cdot \vec{x}) \frac{\exp \left[G^{2} L_{x} L_{y} / 4 \pi\right]}{G^{2} L_{x} L_{y} / 4 \pi}\right\} .
\end{aligned}
$$


These forces are most efficiently calculated together with the energy (in one subroutine).

We now explain the algorithm by which the formation energies of the various types of defect lines are determined. We start with an initial configuration $\left\{\vec{x}^{(0)}(i)\right\}$ which, after relaxation, will contain the desired defect. This is easily achieved by adding or removing lines from the perfect hexagonal lattice. This procedure leads to the defect energies at constant lattice constant, and hence must be corrected as in Eq. (3.38) to produce energies at constant vortex density. The corresponding initial configuration for a vacancy and a variety of other defects are shown in Fig. 6.

The lattice relaxation process was performed by standard methods adapted from molecular dynamics simulations [40]. For advancing the positions and velocities of the flux lines we implemented an artificial dynamics via a leapfrog algorithm

$$
\begin{gathered}
\vec{v}_{\ell}\left(t+\frac{1}{2} \delta t\right)=\vec{v}_{\ell}\left(t-\frac{1}{2} \delta t\right)+\delta t \vec{a}_{\ell}(t) \\
\vec{x}_{\ell}(t+\delta t)=\vec{x}_{\ell}(t)+\delta t \vec{v}_{\ell}\left(t+\frac{1}{2} \delta t\right)
\end{gathered}
$$

The acceleration $\vec{a}_{\ell}=\vec{f}_{\ell} / m$ of the $\ell$ th flux line is calculated from the forces $\vec{f}_{\ell}=\sum_{\ell^{\prime} \neq \ell} \vec{F}_{\ell \ell^{\prime}}$. In each step of the iteration we calculate the kinetic and potential energy, and the forces using Eqs. (3.50), (3.51) and (3.55). This procedure is repeated until an equilibrium configuration is reached. The "mass" $m$ and the time step $\delta t$ were chosen to accelerate the convergence to equilibrium. Equilibrium here means that the forces on the flux lines become zero. Hence the method is capable of finding not only minima but also some saddle point configurations, at least for initial states with high symmetry.

In order to test the accuracy of our method we calculated the energy of a perfect flux line lattice with $N=30 \times t^{2}$ particles for $t=1,2, \ldots, 7$, and compared them with the exact result Eq. (3.52). Our results are summarized in Table 2. The relative difference is less than $10^{-5}$ and can mainly be attributed to the inaccuracy in the numerical approximation we have used for the exponential integral function [41].

Starting from the initial configurations shown in Fig. 6 we have determined the relaxed configurations using the algorithm described by Eqs. (3.56) and (3.57) for various system sizes. From this analysis we can extrapolate to the defect formation energies of an infinite system. 
For the vacancy we find that starting from the initial configuration in Fig. 6, which has the six-fold symmetry of the hexagonal lattice, the lattice first relaxes into a saddle point configuration, which possesses the full symmetry of the lattice. This configuration, however, is unstable with respect to a compression along one of the three axis connecting the nearest neighbors at the vacancy. It finally relaxes into a configuration of lower symmetry (see Fig. 7). Note, that there are three equivalent orientations of this relaxed vacancy configuration. In Table 3 we summarize the formation energies of the stable and saddle point configuration of the vacancy for different system sizes.

The edge interstitial relaxes starting from the initial configuration in Fig. 6 into a saddle point configuration shown in Fig. 8. From the numerical simulation we find that this configuration is unstable with respect to small amplitude "buckling" perpendicular to the edge. The edge interstitial relaxes into a "buckled configuration", which is identical to the relaxed centered interstitial configuration, also shown in Fig. 8. These results are analogous to findings by Cockayne and Elser for the two-dimensional Wigner crystal [25], where the edge interstitial is also unstable with respect to "buckling" perpendicular to the edge of the triangle. However, the time required for this relaxation process is much larger than the relaxation time from an initial edge interstitial configuration constrained by symmetry to go to a final edge state. Thus, the interstitial appears to occupy a very flat minimum in configuration space.

In Table 3 we have listed the defect energies for a vacancy and several types of interstitials for various system sizes. Whereas there is a clear energy gap between vacancies and interstitials, the interstitial energies are all very close. The energy differences are less than $1 \%$ ! The system size dependence for the centered edge, and split centered interstitials and two types of vacancies are shown in Figs. 9a and 9b, respectively.

The lattice conformations resulting from relaxing an edge interstitial and a split edge interstitial initial configuration (constrained now not to buckle as in Fig. 8) are shown in Fig. 10 (also shown by filled circles is the perfect hexagonal lattice). As can be inferred from this figure the configurations essentially differ only by a parallel shift along the edge of the triangle. Because the energies are quite close (see Table 3) we conclude that gliding of this type of defect along the direction defined by the edge of the triangle (in the absence of buckling) must be a low energy excitation. The barrier for this motion is presumably of the order of the difference in energy of those configurations, i.e. $\Delta_{\text {glide }} \approx 10^{-5} E_{0}$ ! After 
very long relaxation times both types of initial conformation, i.e., edge and split edge interstitial, may in fact finally relax into the same final configuration.

We conclude that interstitials, rather than vacancies, are clearly favored at high fields and that the centered interstitial is the most likely candidate for producing a supersolid in this regime. Since the differences between the energies of the various interstitials are so small, an interstitial will have substantial extra entropy, lowering its free energy even further.

\subsection{Interaction between Point Defects}

Following Cockayne and Elser [25], we can use defect energies for different system sizes to infer the distance dependence of the interaction energies. As explained at the beginning of section 3.4, periodic boundary conditions always yield a rectangular superlattice of defects. Since we are changing only the size of the big box $\left(L_{x}, L_{y}\right)$ and not its shape, the system size dependence of the formation energy should scale the same way as the radial dependence of the interaction between two single defects for large $L_{x}$ and $L_{y}$.

From the system size dependence of the centered and edge interstitial energies, plotted in Fig. 9a, one can infer that both defects show an attractive interaction at distances larger than 5 lattice spacings. Over the range studied $5 \leq r \leq 35$ the interaction approximately scales as $r^{-1}$ for centered interstitials and as $r^{-2}$ for edge interstitials. From a nonlinear least square fit over the limited range we get $E_{\text {int }}^{\mathrm{EI}} \approx 0.0742-0.0787 * N^{-1.09}$ corresponding to $r^{-2.18}$ and $E_{\text {int }}^{\mathrm{CI}} \approx 0.0732-0.0078 * N^{-0.61}$ corresponding to $r^{-1.22}$ for the edge and centered interstitial,respectively. As we shall see, these are definitely not the correct asymptotic long distance behaviors.

We have also analyzed numerically the distance dependence of the interaction between two centered interstitials at smaller distances. In order to calculate this energy we have started with an initial configuration, that contains two centered interstitials at a distance $d$ (measured in units of the lattice constant $a$ ) in a perfect hexagonal lattice containing $N=480$ flux lines. The interaction energy is found by subtracting from the resulting relaxation energy the energy of two isolated single centered interstitials. The results for two different directions are displayed in Fig. 11a. If the vector connecting the two centered interstitials points along one of the unit vectors of the primitive cell of the hexagonal lattice, the interaction is attractive up to $d \approx 3 a$ and becomes repulsive for larger distances. 
In the direction perpendicular to one of the unit cell vectors we find a minimum in the interaction potential at a distance of approximately two lattice vectors. (Note that distances in Figs. 11 are the distances between the defects in the initial configuration). The system size difference discussed earlier indicates that, roughly, some angular average of the interaction is attractive for $r>5 a$.

In Fig. 9b we have plotted the system size dependence of the symmetric and "crushed" vacancy configurations. Whereas for a symmetric vacancy configuration the formation energy increases with increasing number of flux lines $N$, it decreases for the "crushed" vacancy configuration. From a nonlinear least square fit to the data in Fig. 9b we find $E_{\mathrm{int}}^{\mathrm{V} 2} \approx 0.1076-0.312 * N^{-1.15}$ corresponding to $r^{-2.3}$ and $E_{\mathrm{int}}^{\mathrm{V} 6} \approx 0.125+0.0576 * N^{-0.64}$ corresponding to $r^{-1.28}$ over the limited range $5 \leq r \leq 35$ for the symmetric and "crushed" vacancy, respectively. Hence we conclude that symmetric vacancies appear to have an attractive interaction at relatively long distances while "crushed" vacancies, which have the lower formation energy repel each other at comparable distances.

For smaller distances we have performed calculations analogous to those for the centered interstitial. The results for the stable "crushed" vacancy (V2) for the interaction along (solid line) and perpendicular (dashed line) to an edge of the unit cell are shown in Fig. 11.b. Whereas the interaction energy is attractive for all distances less than $11 a$ along the directions perpendicular to the edges of the unit cell, it is attractive for small distances and becomes repulsive for distances larger than $d \approx 5 a$ for the interaction along the edge of the unit cell. This has to be compared with the roughly angular averaged attractive interaction for $d>5 a$ obtained from the finite size analysis.

It is not possible to study the interaction between symmetric vacancies at short distances. This is because the anisotropy of the stresses induced by the interactions causes the vacancies to deform to the anisotropic crushed vacancy configuration which has lower energy. The symmetry axis of the resulting crushed vacancies is parallel to their separtion vector.

It is instructive to compare these results with those obtained from linear elasticity theory which should be valid for very large separations. It can be shown that the $\ln r$ interactions between unrelaxed defects will be completely screened by the relaxation of the other vortex lines. This is already evident in the calculations in section 3.4 for a single 
vacancy: the vortex lines far from the vacancy relax just so as to cancel the overall "charge" of the vacancy. The long distance interactions between defects thus have the same form as for short-range interactions. These depend on the symmetries of the defects involved.

For defects with three- or six-fold symmetry - the symmetric vacancies and centered interstitials - the interactions are exponentially small in the absence of anisotropy of the elastic interactions with the anisotropy associated with the six-fold symmetry [which appear at order $q^{4}$ in the elastic matrix $C^{\alpha \beta}(\vec{q})$ of Eq. (3.17)], the interactions will decay as $\cos 6 \theta / r^{4}$ with $\theta$ the angle between a lattice vector and the inter-defect separation vector. The sum over all the inter-defect interactions with the periodic boundary conditions used in the numerical computations will thus almost cancel as the systems used are almost square with $L_{y}^{2} / L_{x}^{2}=25 / 27$. The resulting asymptotic $L$ dependence of the vacancy energy should thus have a leading $1 / L^{4}$ term with a small coefficient whose sign depends on details of the $q$ dependence of the elastic matrix and the stresses induced by the vacancy which we have not calculated.

The interactions between defects with only a two-fold symmetry axis-edge and splitcentered interstitials and crushed vacancies are longer range since they would decay slowly even in an isotropic elastic medium. The interactions will have two comparable contributions at long distances.

$$
E_{\mathrm{int}} \approx \frac{K_{2} \cos 2 \phi}{r^{2}}+\frac{K_{4} \cos 4 \phi}{r^{2}}
$$

where $\phi$ is the angle between the symmetry axis of the defect and the separation vector. The coefficient $K_{4}$ is always positive (i.e., repulsive) while the sign of $K_{2}$ depends on the stresses induced by the defects. In the almost square system with periodic boundary conditions used in the numerical calculations, the $\cos 2 \phi$ term will almost cancel and the leading size dependence of the defect energies will be $1 / L^{2}$ with a sign $\cos 4 \alpha$ which depends on the cosine of the angle $\alpha$ between the $x$ direction and the symmetry axis of the defects. At long distances, however, the actual sign of the interaction between two isolated defects will depend on the direction of the defect separation in a different way if $\left|K_{2}\right|>K_{4}$.

The apparent distance dependence seen in the numerical calculations is a fit over limited range of distance and presumably indicates that the asymptotic behavior discussed above has not been reached. 


\section{EQUILIBRIUM DESCRIPTION OF THE SUPERSOLID PHASE}

We now assume that centered interstitials are energetically preferred (as found for zero temprature in Sec. 3), and study the low lying excitations via a simple tight-binding model. We find a more quantitative estimate of the transition temperature $T_{d}$, discuss the nature of the transitions at $T_{d}$ and $T_{m}$ and compare the properties of the solid, supersolid and liquid phases.

\subsection{Tight-binding Model and the Transition Temperature}

As discussed in Sec. 2, the defect proliferation temperature is determined by the large $L$ behavior of the partition function (1.2). Equation (1.2) may also be written as an integral over a quantum mechanical matrix element analogous to Eq. (2.6),

$$
\mathcal{Z}_{d}=\int d^{2} \vec{r}_{i} \int d^{2} \vec{r}_{j}\left\langle\vec{r}_{f}\left|e^{-\mathcal{H}_{d} L / T}\right| \vec{r}_{i}\right\rangle,
$$

where the integral is over entry and exit points $\vec{r}_{i}$ and $\vec{r}_{j}$ for the interstitial and $\mathcal{H}_{d}$ is an effective quantum Hamiltonian describing the transfer matrix. We adopt a lowtemperature perspective and use for $\mathcal{H}_{d}$ a tight-binding model which assumes that the centered interstitial sits at the sites of a simple honeycomb lattice. As shown in Fig. 13, the honeycomb lattice may be viewed as two interpenetrating triangular lattices with sites $\left\{\vec{r}_{A}\right\}$ and $\left\{\vec{r}_{B}\right\}$, connected by displacement vectors $\left\{\vec{\delta}_{i}, i=1,2,3\right\}$ with

$$
\vec{\delta}_{1}=b(0,1), \quad \vec{\delta}_{2}=b\left(\frac{\sqrt{3}}{2},-\frac{1}{2}\right), \quad \vec{\delta}_{3}=b\left(-\frac{\sqrt{3}}{2},-\frac{1}{2}\right)
$$

and $b=\frac{\sqrt{3}}{3} a$ with $a_{0}$ the triangular lattice constant.

The interstitial can "tunnel" from one site to another by passing through an edge (interstitial) state, which according to Table 3, has an energy nearly degenerate with the centered interstitial. The tight-binding Hamiltonian is then 


$$
\begin{aligned}
\mathcal{H}_{d}=u_{0} & {\left[\sum_{\vec{r}_{A}}\left|\vec{r}_{A}\right\rangle\left\langle\vec{r}_{A}\left|+\sum_{\vec{r}_{B}}\right| \vec{r}_{B}\right\rangle\left\langle\vec{r}_{B}\right|\right] } \\
& -t\left[\sum_{\vec{r}_{A}, \vec{\delta}_{A}}\left|\vec{r}_{A}\right\rangle\left\langle\vec{r}_{A}+\vec{\delta}_{A}\left|+\sum_{\vec{r}_{B}, \vec{\delta}_{B}}\right| \vec{r}_{B}\right\rangle\left\langle r_{B}+\vec{\delta}_{B}\right|\right]
\end{aligned}
$$

where $\left|\vec{r}_{A}\right\rangle$ and $\left|\vec{r}_{B}\right\rangle$ are normalized states on the two sublattices, $\left\{\vec{\delta}_{A}\right\}=\left\{\vec{\delta}_{i}\right\}$ and $\left\{\vec{\delta}_{B}\right\}=$ $\left\{-\vec{\delta}_{i}\right\}$. Here $\vec{u}_{0}$ is a site energy, and $t$ is a tunnelling matrix element.

The eigenvectors of (4.3) are the linear combinations $\psi_{ \pm}(\vec{k}) \equiv \frac{1}{\sqrt{2}}(|\vec{k}, A\rangle \pm|\vec{k}, B\rangle)$ of normalized plane wave states,

$$
|\vec{k}, A\rangle=\frac{1}{\sqrt{N_{0}}} \sum_{\vec{r}_{A}} e^{i \vec{k} \cdot \vec{r}_{A}}\left|\vec{r}_{A}\right\rangle, \quad|\vec{k}, B\rangle=\frac{1}{\sqrt{N_{0}}} \sum_{\vec{r}_{B}} e^{i \vec{k} \cdot \vec{r}_{B}}\left|\vec{r}_{B}\right\rangle,
$$

where $N_{0}$ is the number sites in one sublattice and $\vec{k}$ is confined to a hexagonal Brillouin zone. The eigenvalues consist of two bands,

$$
\epsilon_{ \pm}(\vec{k})=u_{0} \mp|t| \sqrt{3+2\left[\cos \left(\vec{k} \cdot \vec{\delta}_{1}\right)+\cos \left(\vec{k} \cdot \vec{\delta}_{2}\right)+\cos \left(\vec{k} \cdot \vec{\delta}_{3}\right)\right]}
$$

which are nondegenerate except at the zone corners. The lowest eigenvalue occurs in $\varepsilon_{+}(k)$ at $\vec{k}=0$,

$$
E_{0}(T)=u_{0}-3|t|,
$$

and dominates the partition sum (4.1) in the limit $L \rightarrow \infty$. The defect unbinding temperature $T_{d}$ is determined by the condition $E_{0}\left(T_{d}\right)=0$. To proceed further, we need to determine $u_{0}$ and $t$.

We assume the tunnelling process between sites of the honeycomb lattice can be modeled by a one-dimensional quartic potential in the coordinate $x$ connecting two honeycomb lattice sites,

$$
V(x)=\frac{\tilde{\epsilon}_{d} \omega^{2}}{2 b^{2}}\left(x-\frac{1}{2} b\right)^{2}\left(x+\frac{1}{2} b\right)^{2}
$$

where the potential vanishes at the lattice sites. The frequency $\omega$ is fixed by equating the maximum to $\Delta \epsilon>0$, the energy difference between the edge and centered interstitials in Table 2,

$$
\omega=4 \sqrt{2} \sqrt{\frac{\Delta \epsilon}{\tilde{\epsilon}_{d}}} \frac{1}{b}
$$


A standard quantum mechanical calculation [43] then gives

$$
E_{ \pm}=T \omega\left[\frac{1}{2} \pm 2 \sqrt{\frac{3}{2 \pi}}\left(\frac{S_{0}}{T}\right)^{1 / 2} e^{-S_{0} / T}\right]
$$

for the two lowest lying levels, with

$$
S_{0}=\frac{1}{6} \tilde{\varepsilon}_{d} \omega b^{2}
$$

The WKB exponential factor $S_{0}$ is just the "kink energy" of a defect with stiffness $\tilde{\epsilon}_{d}$ which moves between the two sites as a function of $z$ in the path integral (1.2). Upon identifying the splitting in (4.9) with $t$ in Eq. (4.3), we find

$$
t=2 \sqrt{\frac{3}{2 \pi}} \omega\left(T S_{0}\right)^{1 / 2} e^{-S_{0} / T}
$$

To a zeroeth approximation, we have $u_{0} \equiv \varepsilon_{d}$, the energy of the centered interstitial computed in Sec. 3. In principle, $u_{0}$ should be corrected by the zero point energy of a twodimensional quantum oscillator, i.e., by twice the first term of Eq. (4.9). This represents the entropy of harmonic fluctuations of the defect. There is also, however, a negative contribution of this form from the entropy of the flux line lattice itself [25] which should have approximately the same magnitude. We assume for simplicity here that these two contributions simply cancel.

It is convenient now to set

$$
u_{0}=\alpha \Delta \epsilon,
$$

where according to Table $3, \alpha \approx 70$. Upon using Eqs. (4.11) and (4.10), we find that the condition $E_{0}\left(T_{d}\right)=0$ takes the form

$$
\alpha\left(\frac{S_{0}}{T_{d}}\right)^{1 / 2}=32 \sqrt{\frac{3}{2 \pi}} e^{-S_{0} / T_{d}}
$$

which is solved by $S_{0} / T_{d}=0.085$. The assumption $\tilde{\varepsilon}_{d} \approx \tilde{\varepsilon}_{1}$, then leads to

$$
T_{d}=0.30 \sqrt{\varepsilon_{0} \tilde{\varepsilon}_{1}} a_{0}
$$

i.e., Eq. (2.24) with $c_{3}=0.30$. The substitution $c_{L}=0.15-0.30$ in Eq. (2.11), however, shows that the melting temperature $T_{m}$ is significantly (about an order of magnitude) 
smaller than our estimate of $T_{d}$ in the regime $B_{\mathrm{c} 1}<B<B_{\times}$. Evaluation of the high field formula Eq. (2.26) at $B=B_{\times}$is only slightly more encouraging: Using Table 3 for the centered interstitial energy leads to $T_{d}=0.079 \varepsilon_{0} d_{0}$, while the Lindemann criteria at $B=B_{\times}$gives $T_{m}=0.02-0.09 \varepsilon_{0} d_{0} \sim T_{d}$ [44]. We conclude that the supersolid probably only exists for $B>B_{\times}$, as indicated in Fig. 5 .

Of course, our estimate for $S_{0} / T_{d}$ is so small that it casts doubt on the validity of the tight-binding model and the WKB approximation, which are only strictly correct at low temperatures. More accurate band structure estimates of $T_{d}$ for $B<B_{\times}$(including $\left.B<B_{\mathrm{c} 1}\right)$ would certainly be of interest.

\subsection{Nature of the Transition at $T_{d}$}

As discussed above, a transition to a supersolid below the equilibrium melting temperature of the flux crystal becomes possible when $B>B_{\times}$. Once the defects proliferate, interactions between them become important, as in the closely related problem of vortex penetration just above $\mathrm{H}_{\mathrm{c} 1}$. To model this process, we use a continuum coherent state path integral representation of the partition function, similar to one used for flux lines near $\mathrm{H}_{\mathrm{c} 1}$ [7]. The details of the lattice of preferred sites are neglected, although these could easily be taken into account if necessary. The grand canonical partition function describing the interstitial degrees of freedom then reads

$$
\mathcal{Z}_{g r}=\int \mathcal{D} \psi_{i} \mathcal{D} \psi^{*} e^{-S\left[\psi, \psi^{*}\right]}
$$

with

$$
S\left[\psi, \psi^{*}\right]=\int d^{2} r d z\left[\psi_{i}^{*}\left(\partial_{z}-\frac{T}{2 \tilde{\varepsilon}_{d}} \nabla_{\perp}^{2}\right) \psi_{i}+r\left|\psi_{i}\right|^{2}+u\left|\psi_{i}\right|^{4}+\cdots\right]
$$

Here $\psi_{i}(\vec{r}, z)$ and $\psi_{i}^{*}(\vec{r}, z)$ represent interstitial line creation and annihilation operators, and the $r \propto\left(T_{d}-T\right)$ is the defect chemical potential. The areal density of interstitials $n_{i}$ is given by

$$
n_{i}=\left|\psi_{i}(r, z)\right|^{2}
$$

and the couplings $u$ and $v$ represent the effect of interactions.

The nature of the transition to the supersolid which occurs with decreasing $r$ depends crucially on the sign of the quartic coupling in Eq. (4.15). If $u>0$, then a continuous transition results, and $n_{d} \propto\left(T-T_{d}\right)$ up to logarithmic corrections. Both the coefficient and the 
logarithmic correction can be calculated as in [7]. If $u<0$, the transition to the supersolid becomes first order. A first-order transition is possible because the microscopic two-body interaction between centered interstitials (represented by $u|\psi(\vec{r}, z)|^{4}$ in Eq. (4.15)) is attractive, at least at large distances; see Section 3. The unbinding temperature $T_{d}<T_{m}$ is probably too low to allow for a significant thermal renormalization of $u$, which could in principle be driven positive by entropic effects. The first-order transition described by (4.15) with $u$ negative is discussed in Ref. 45.

A finite density of proliferating defects is detectable, at least in principle, via a neutron diffraction experiment which precisely determines the temperature dependent magnitude of the six smallest reciprocal lattice vector $\{\vec{G}(T)\}$ of the Abrikosov flux array for fixed magnetic field $\vec{B}$. Note that $\vec{B}$ will also be, in general, temperature-dependent for fixed external field $\vec{H}$. The number of unit cells per unit area $n_{c}$ is related to $G$ according to

$$
n_{c}(T)=\sqrt{3} G^{2}(T) / 8 \pi^{2}
$$

If vacancies or interstitials only exist in small closed loop pairs, as in Fig. 1b, $n_{c}$ must be exactly equal to the areal density of vortices, $n_{c}=n_{0}=B / \phi_{0}$. Above the proliferation temperature $T_{d}$, interstitials dominate over vacancies, and we have

$$
\begin{aligned}
n_{i}(T) & =\left\langle\left|\psi_{i}(\vec{r}, z)\right|^{2}\right\rangle \\
& =n_{0}-n_{c}(T)>0
\end{aligned}
$$

A plot of $n_{c}(T) / n_{0}$ is thus a direct measure of the density of defects, with $n_{c}(T) / n_{0} \equiv 1$ for $T<T_{d}$, and $n_{c}(T) / n_{0}<1$ when $T>T_{d}$.

A nonzero defect order parameter $\left\langle\psi_{i}(r, z)\right\rangle$ necessarily implies that the boson order parameter (1.6) is nonvanishing, because wandering vacancies or interstitial defects catalyze enhanced entanglement of the underlying vortex crystal. The vortices are thus simultaneously crystalline and entangled when $T_{d}<T<T_{m}$, as discussed in the Introduction. Once the equilibrium concentration of one type of defect is nonzero, all defects will proliferate in at least small concentrations. Consider, in particular, the process shown in Fig. 1b, the creation of vacancy-interstitial pairs. This can be modeled by adding terms to the action (4.15) as follows:

$$
S \rightarrow S+\int d^{2} r d z\left[\psi_{v}^{*}\left(\partial_{z}-\frac{T}{2 \tilde{\varepsilon}_{d}} \nabla_{\perp}^{2}\right) \psi_{v}+r_{v}\left|\psi_{v}\right|^{2}+g\left(\psi_{i} \psi_{v}+\psi_{i}^{*} \psi_{v}^{*}\right)\right]
$$


The first two terms are the vacancy propagator and chemical potential, while the last allows pair creation and destruction with probability proportional to $g$ [46]. Because the vacancies are unfavorable relative to interstitials, $r_{v}$ will remain positive just above $T_{d}$. We see, however, that a nonzero $\left\langle\psi_{i}(\vec{r}, z)\right\rangle$ acts like an ordering field on $\psi_{v}(r, z)$, i.e., $\left\langle\psi_{v}(\vec{r}, z)\right\rangle \neq 0$ for $T>T_{d}$. In more physical terms, vacancies can appear because their unfavorable energy in isolation is compensated by nearby thermally created interstitials.

We also mention an exotic type of supersolid which is possible at least in principle: Suppose that the split interstitial, or some similar defect had the lowest energy. The three distinct orientations of this defect within its hexagonal cell represent an internal degree of freedom for the corresponding "boson" world lines. Should such "ribbon-like" defects proliferate in the solid, the resulting fluid of lines would be a "quantum rotator" liquid, with the same potential broken symmetries as a quantum three-state Potts model when interline interactions are taken into account.

\subsection{Nature of the Transition at $T_{m}$}

It is important to distinguish between Type I and Type II melting into a liquid phase, according to whether vacancy and interstitial defects have already proliferated - see Fig. 4. Consider the standard Landau argument for a first-order transition starting from the flux liquid state: Provided fluctuations suppress crystallization well below the mean field $\mathrm{H}_{\mathrm{c} 2}$, one can expand the local BCS condensate density in Fourier components of the incipient density wave,

$$
\left\langle\left|\psi_{B C S}(\vec{r}, z)\right|^{2}\right\rangle=\rho_{0}+\sum_{\vec{G}} \rho_{\vec{G}} e^{i \vec{G} \cdot \vec{r}}
$$

where the $\{\vec{G}\}$ are reciprocal lattice vectors in a plane perpendicular to $\hat{z}$. The free-energy difference $\delta \mathcal{F}$ between the liquid and crystalline phases can then be expressed as a Taylor series in the order parameters $\left\{\rho_{\vec{G}}\right\}$,

$$
\delta \mathcal{F}=\frac{1}{2} r_{G} \sum_{j=1}^{6}\left|\rho_{\vec{G}_{j}}\right|^{2}+w \sum_{\vec{G}_{i}+\vec{G}_{j}+\vec{G}_{k}=0} \rho_{\vec{G}_{i}} \rho_{\vec{G}_{j}} \rho_{\vec{G}_{k}}+\cdots
$$

where we have included only the first ring of six smallest $\vec{G}$ 's. The crucial element is the third-order term allowed by the symmetry of a triangular lattice, which leads to a firstorder transition within this mean field theory [2]. The magnitude of the smallest $G$ 's in 
Eq. (4.20) would be completely determined by the magnetic field for Type I melting, but would depend on the incipient density of vacancies or interstitials in the Type II case, as discussed in Sec. 4.2 .

Tesanovic [42] has suggested that when the freezing transition approaches $H_{c 2}$, as it will at low temperatures or if the fluctuations are weak, the transition will be to a charge density wave state with a wavevector not simply related to the magnetic field. Such a state is similar to the "supersolid" phase discussed here, and presumably evolves continuously into it as the melting field falls well below $H_{c 2}$. The line of supersolid phase transitions may also be related to the solid-phase transition suggested by Glazman and Koshelev [24], above which phase coherence is lost in the $z$ direction. See Sec. 4.4.

Note that generically, if a melting transition is weakly first order, it is likely to be to an incommensurate solid phase as the wave-vector-dependent $r_{G}$ in Eq. (4.21) will in general not have its minimum at any particularly simple wavevector.

The distinction between Type I and Type II behavior also affects dislocation mediated melting theories, which start from the ordered phase just below $T_{m}$. Reference 8 , for example, studies effects of dislocation loops which are confined to the plane spanned by their Burger's vector and the $z$-axis. This restriction implicitly assumes Type I melting, i.e., that no vacancies or interstitials are present at long wavelengths. The absence of vacancy or interstitial lines means that only "glide" motions of dislocation lines are allowed along the time-like $z$ axis, which is equivalent to a planarity restriction for the three-dimensional vortex loops. A small concentration of proliferating vacancy or interstitial lines would allow "climb-like" distortions of a vortex loop, as the loop absorbs or emits these defects. Type II dislocation mediated melting would thus involve arbitrary nonplanar dislocation loop configurations, in contrast to the planar loops associated with Type I melting.

\subsection{Transport Properties}

Finally, we compare briefly the resistive properties of the supersolid, crystal and vortex fluid phases.

In the absence of pinning by random inpurities, all the above phases dissipate currents perpendicular to the macroscopic magnetic field since the vortex lines can move freely provided - in the solid-the motion is uniform. In contrast, the vortex crystal is a linear 
superconductor for currents parallel to the $z$ direction. Concommitantly, it can screen additional magnetic fields normal to the $z$ direction. This will not occur in a semi-infinite system with a planar surface since the magnetic fields can just rotate. But in a cylinder with the vortex parallel to the axis, a small additional azimuthal magnetic field will be expelled, decaying exponentially in a layer with thickness given by an effective penetration length [48].

Even in the crystal phase, finite currents parallel to $\hat{z}$ will be dissipated by nonlinear nucleation of vorticity normal to $\hat{z}$; the details of this process in bulk samples have not, to our knowledge, been analyzed.

It is interesting to note that while the vortex crystal is very anisotropic in response to uniform currents, it appears much more isotropic in its linear confinement of magnetic monopoles as discussed in the Introduction.

Both the vortex supersolid and vortex fluid phases will respond like normal metals to currents parallel to $\hat{z}$, although there will be additional nonlinear dispersion and nonlocal effects [49]. The linear resistivity may also be extremely anisotropic if the vortex lines are rather straight. In the supersolid phase, the dissipation will be dominated by the fluid of interstitial (or vacancy) lines while the underlying lattice will not move in response to a small current in the $z$ direction.

Pinning by random impurities, oxygen vacancies or other defects strongly affects the resistive properties of the mixed state of superconductors. If the pinning is very weak, the thermodynamic properties will be little affected but the long-range translational order of both the crystal and supersolid phases will be destroyed on long distances, resulting in a large but finite positional correlation length. The crystal phase may be replaced in its entirety by a truly superconducting vortex glass phase [12] with vanishing linear resistivity. In contrast, the vortex fluid phase is not much affected by weak pinning. Because the defects are fluid-like in the supersolid phase, they also will not be strongly affected by weak pinning, provided they are sufficiently dense [50]. The resulting phase will then still be metallic with nonzero resistivity. Thus, it appears likely that the vortex-glass phase transition for weak pinning should occur at what was the defect proliferation transition, $T_{d}$, in the pure system. If the vortex lattice melting is Type $I$, then the putative vortex-glass transition should probably occur at the $T_{m}$ of the pure system. 
The nonlinear response of the weakly pinned supersolid will involve motion of both the underlying lattice, including plastic flow involving dislocations, as well as vacancies and interstitials. Thus, even if the defect lines themselves are pinned, and the supersolid is in a vortex glass phase, the nonlinear response might distinguish it from a weakly pinned crystal.

In the presence of sufficiently strong pinning, both equilibrium phase transitionsdefect proliferation and melting — will be destroyed. The vortex glass transition, if it exists at finite temperatures, will then become second order with the resistance vanishing continuously as the temperature is decreased. In this case, the distinctions between the low temperature solid phases will disappear as the extent of any crystaline order will be very short range. There are, however, several caveats. Firstly, it is likely that hexatic bondorientational order can persist out to much longer distances than positional order [51], and perhaps even to infinite distances so that distinct hexatic and vortex glass transitions could occur. Another possibility is that more than one type of vortex glass phase could exist, one with residual shear elasticity, as hypothesized by Feigel'man et al. [53] and one without shear elasticity as discussed by Fisher, Fisher and Huse [12]. Which of these phases actually can exist even in principle is still very unclear. This complex set of possibilities illustrates the subtleties once random point pinning is taken into account.

As mentioned earlier, the basic phase boundaries may well, except for strong pinning, be determined by the properties of the pure vortex systems analyzed in this paper. Thus, a more quantitative analysis of the phase diagram in the pure system is certainly merited.

Acknowledgements We have benefited from conversations with D.J. Bishop, V. Elser, D.A. Huse, and C.M. Murray. This work was supported by National Science Foundation, through Grants No. DMR91-15491 and DMR91-06237 and through the Harvard Materials Research Laboratory. The work of Erwin Frey has been supported by the Deutsche Forschungsgemeinschaft (DFG) under Contracts No. Fr. 850/2-1,2. 


\section{Appendix A: Calculation of the Dynamic Matrix in the Limit $\lambda_{\perp} \rightarrow \infty$.}

In this Appendix we describe the details of the calculation of the dynamic matrix. Starting from Eq. (3.16) the central quantity to calculate is

$$
I(\vec{x} / \lambda)=\sum_{l} e^{-i \vec{q} \cdot \vec{x}(l)} K_{0}(|\vec{x}(l)-\vec{x}| / \lambda)-K_{0}(|\vec{x}| / \lambda),
$$

where we set $\lambda_{\perp} \equiv \lambda$ in what follows. Upon using the integral representation of the Bessel function $K_{0}$

$$
K_{0}(x)=\frac{1}{2} \int_{0}^{\infty} d \tau \tau^{-1} e^{-\tau} \exp \left[-\frac{x^{2}}{4 \tau \lambda^{2}}\right],
$$

and Ewald's generalized theta-function transform [39]

$$
\sum_{l} e^{-i \vec{q} \cdot \vec{x}(l)} \exp \left[-|x(l)-x|^{2} t\right]=\frac{\pi}{a_{c} t} \sum_{\vec{G}} e^{-i(\vec{q}+\vec{G}) \cdot \vec{x}} \exp \left[-|\vec{q}+\vec{G}|^{2} / 4 t\right],
$$

where $a_{c}$ is the volume of the unit cell, one can rewrite the first term in Eq. (A1) as

$$
\begin{aligned}
I_{1}(\vec{x} / \lambda) & =\sum_{l} e^{-i \vec{q} \cdot \vec{x}(l)} K_{0}\left(|\vec{x}(l)-\vec{x}| / \lambda^{2}\right) \\
& =\frac{1}{2} \int_{0}^{\infty} d \tau \tau^{-1} e^{-\tau} \sum_{l} e^{-i \vec{q} \cdot \vec{x}(l)} \exp \left[-\frac{|\vec{x}(l)-\vec{x}|^{2}}{4 \tau \lambda^{2}}\right] \\
& =\frac{2 \pi}{a_{c}} \lambda^{2} \int_{0}^{\infty} d \tau e^{-\tau} \sum_{\vec{G}} e^{-i(\vec{q}+\vec{G}) \cdot \vec{x}} \exp \left[-\tau \lambda^{2}|\vec{q}+\vec{G}|^{2}\right]
\end{aligned}
$$

Now we split the $\tau$-integration by introducing an arbitrary Ewald split-parameter $\epsilon$

$$
\begin{aligned}
I_{1}(\vec{x} / \lambda)= & \frac{1}{2} \int_{0}^{\epsilon} d \tau \tau^{-1} e^{-\tau} \sum_{l} e^{-i \vec{q} \cdot \vec{x}(l)} \exp \left[-\frac{|x(l)-x|^{2}}{4 \tau \lambda^{2}}\right] \\
& +\frac{2 \pi}{a_{c}} \lambda^{2} \sum_{\vec{G}} e^{-i(\vec{q}+\vec{G}) \cdot \vec{x}} \frac{e^{-\epsilon\left(\lambda^{2}|\vec{q}+\vec{G}|^{2}+1\right)}}{1+\lambda^{2}\left|\vec{q}_{\vec{G}}\right|^{2}}
\end{aligned}
$$

where we have already performed the $\tau$-integral in the second term.

We are interested in the limit of large London penetration depth $\lambda$. Upon choosing $\epsilon \sim \lambda^{-2}$ we take the limit $\lambda \rightarrow \infty$ with $\epsilon \lambda^{2}$ staying finite. Then one gets for the second term in Eq. (A.5)

$$
\frac{2 \pi}{a_{c}} \lambda^{2} \sum_{\vec{G}} e^{-i(\vec{q}+\vec{G}) \cdot \vec{x}} \frac{e^{-\epsilon \lambda^{2}|\vec{q}+\vec{G}|^{2}}}{1+\lambda^{2}\left|\vec{q}_{\vec{G}}\right|^{2}} .
$$


For the first term we make the substitution $y=|\vec{x}(l)-\vec{x}|^{2} /\left(4 \tau \lambda^{2}\right)$ and get

$$
\frac{1}{2} \int_{a}^{\infty} \frac{d y}{y} \sum_{l} \exp \left[-\frac{|\vec{x}(l)-\vec{x}|^{2}}{4 y \lambda^{2}}\right] e^{-y} e^{-i \vec{q} \cdot \vec{x}(l)}
$$

In the limit $\lambda \rightarrow \infty$ we get $\exp \left[-\frac{|\vec{x}(l)-\vec{x}|^{2}}{4 y \lambda^{2}}\right] \rightarrow 1$ since $y$ is bounded from below by $a=\frac{1}{4 \epsilon \lambda^{2}}|\vec{x}(l)-\vec{x}|^{2}$. Hence the first term can be written in terms of the exponential integral function which is defined by

$$
E_{1}(x)=-E i(-x)=\int_{x}^{\infty} \frac{d y}{y} e^{-y}
$$

In summary one gets

$$
\begin{aligned}
\lim _{\lambda \rightarrow \infty} I_{1}(\vec{x} / \lambda)= & \frac{1}{2} \sum_{l} e^{-i \vec{q} \cdot \vec{x}(l)} E_{1}\left(\frac{|\vec{x}(l)-\vec{x}|^{2}}{4 \epsilon \lambda^{2}}\right) \\
& +\frac{2 \pi}{a_{c}} \lambda^{2} \sum_{\vec{G}} e^{-i(\vec{q}+\vec{G}) \cdot \vec{x}} \frac{e^{-\epsilon \lambda^{2}|\vec{q}+\vec{G}|^{2}}}{|\vec{q}+\vec{G}|^{2}}
\end{aligned}
$$

In order to get $I(|\vec{x}| / \lambda)$ one has to subtract

$$
K_{0}(x / \lambda)=\frac{1}{2} \int_{0}^{\infty} d \tau \tau^{-1} e^{-\tau} e^{-x^{2} / 4 \lambda \tau} \approx--\ln (x / 2 \lambda)
$$

from $I_{1}(|\vec{x}| / \lambda)$ in the limit $\lambda \rightarrow \infty$. Therefrom one can now calculate $C_{\alpha \beta}$ and expand in powers of the wave vector $\vec{q}$. The result is

$$
C_{\alpha \beta}(\vec{q})=\frac{\phi_{0}^{2}}{8 \pi^{2} \lambda^{2}}\left\{2 \pi \frac{q^{\alpha} q^{\beta}}{q^{2}}+\frac{a_{c}}{8}\left[\delta^{\alpha \beta} q^{2}-2 q^{\alpha} q^{\beta}\right]\right\} .
$$

where we have taken $1 /\left(4 \epsilon \lambda^{2}\right)=\pi / a_{c}$ for the Ewald separation parameter.

\section{Appendix B: Variational Calculation of the Vacancy Formation Energy}

In this appendix we give the details of the variational calculation for the vacancy formation energy. Upon using the long wavelength approximation (3.18) for $C^{\alpha \beta}(\vec{q})$, one finds three contributions to $E_{\mathrm{VR}}^{(2)}$, the vacancy relaxation energy at constant lattice spacing,

$$
E_{\mathrm{VR}}^{(2)}(\{\vec{u}(\ell)\})=2 \epsilon_{0}\left(I_{1}+I_{2}+I_{3}\right)
$$


with

$$
\begin{gathered}
I_{1}=\frac{1}{2} \int \frac{d^{2} q}{(2 \pi)^{2}}(i)^{2} \frac{q^{\alpha}}{q^{2}} \frac{-q^{\beta}}{q^{2}} f^{2}(q)\left\{2 \pi \frac{q^{\alpha} q^{\beta}}{q^{2}}+\frac{a_{c}}{8}\left(\delta^{\alpha \beta} q^{2}-2 q^{\alpha} q^{\beta}\right)\right\} \\
=\frac{1}{2} \int_{0}^{k_{d}} d q q^{-1} f^{2}(q)-\frac{a_{c}}{32 \pi} \int_{0}^{k_{d}} d q q f^{2}(q) \\
I_{2}=-\int_{q} i\left(\frac{2 \pi}{q^{2}}-\frac{a_{c}}{2}\right) q^{\alpha} i \frac{-q^{\alpha}}{q^{2}} f(q) \\
=-\int_{0}^{k_{d}} d q q^{-1} f(q)+\frac{a_{c}}{4 \pi} \int_{0}^{k_{d}} d q q f(q)
\end{gathered}
$$

and

$$
\begin{aligned}
I_{3}= & -\frac{1}{2 a_{c}} \int_{q} \int_{k}(-1)\left\{\frac{2 \pi(q-k)^{\alpha}(q-k)^{\beta}}{(\vec{q}-\vec{k})^{2}}+\frac{a_{c}}{8}\left(\delta^{\alpha \beta}(\vec{q}-\vec{k})^{2}-2(q-k)^{\alpha}(q-k)^{\beta}\right)\right\} \\
& \times(i)^{2} a_{c}^{2} \frac{k^{\alpha}}{k^{2}} f(k) \frac{-q^{\beta}}{q^{2}} f(q) \\
= & \frac{a_{c}}{2} \int_{q} \int_{k} f(q) f(k)\left[2 \pi \frac{\left(\vec{q} \cdot \vec{k}-k^{2}\right)\left(q^{2}-\vec{q} \cdot \vec{k}\right)}{\left(q^{2}-2 \vec{q} \cdot \vec{k}+k^{2}\right) q^{2} k^{2}}+\frac{a_{c}}{8}(\vec{q} \cdot \vec{k})\left(q^{2}-2 \vec{q} \cdot \vec{k}+k^{2}\right) \frac{1}{q^{2} k^{2}}\right. \\
& \left.-\frac{a_{c}}{4}\left(\vec{q} \cdot \vec{k}-k^{2}\right)\left(q^{2}-\vec{q} \cdot \vec{k}\right) \frac{1}{q^{2} k^{2}}\right]
\end{aligned}
$$

where $f(q)=f\left(1+c q+d q^{2}\right)$ and we have approximated the hexagonal Brillouin zone by a circle of equal area with radius $k_{d}=\sqrt{\frac{4 \pi}{a_{c}}}$.

The third contribution can be split into $I_{3}=I_{3 a}+I_{3 b}+I_{3 c}$ where

$$
I_{3 a}=a_{c} \pi \int_{0}^{k_{d}} q d q \int_{0}^{k_{d}} k d k \frac{2 \pi}{(2 \pi)^{4}} \int_{-\pi}^{\pi} d \varphi f(p) f(k) \frac{\left(q k \cos \varphi-k^{2}\right)\left(q^{2}-q k \cos \varphi\right)}{\left(q^{2}-2 q k \cos \varphi+k^{2}\right) q^{2} k^{2}} .
$$

Upon using

$$
F\left(q_{1}, q_{2}\right)=\int_{-\pi}^{\pi} d \varphi \frac{\left(q_{2}^{2}-q_{1} q_{2} \cos \varphi\right)\left(-q_{1}^{2}-q_{1} q_{2} \cos \varphi\right)}{q_{1}^{2}+q_{2}^{2}-2 q_{1} q_{2} \cos \varphi}= \begin{cases}-\pi q_{2}^{2} & \text { for } q_{1}>q_{2} \\ -\pi q_{1}^{2} & \text { for } q_{1}<q_{2}\end{cases}
$$

one arrives at

$$
\begin{aligned}
I_{3 a} & =-\frac{a_{c}}{8 \pi} \int_{0}^{k_{d}} d q \int_{0}^{k_{d}} \frac{f(q) f(k)}{q k} \begin{cases}k^{2} & \text { for } k<q \\
q^{2} & \text { for } k>q\end{cases} \\
& =-\frac{a_{c}}{8 \pi} \int_{0}^{k_{d}} d q\left(\int_{0}^{q} \frac{k}{q} f(q) f(k) d k+\int_{q}^{k_{d}} \frac{q}{k} f(q) f(k) d k\right),
\end{aligned}
$$




$$
\begin{aligned}
I_{3 b} & =\frac{a_{c}^{2}}{16} \int_{0}^{k_{d}} q d q \int_{0}^{k_{d}} k d k \frac{1}{(2 \pi)^{3}} \int_{-\pi}^{\pi} d \varphi \frac{\cos \varphi}{q k} f(p) f(k)\left(q^{2}-2 q k \cos \varphi+k^{2}\right) \\
& =-\frac{a_{c}^{2}}{16(2 \pi)^{2}} \int_{0}^{k_{d}} d q \int_{0}^{k_{d}} d k f(q) f(k) q k, \\
I_{3 c} & =-\frac{a_{c}^{2}}{8} \int_{0}^{k_{d}} q d q \int_{0}^{k_{d}} k d k \frac{1}{(2 \pi)^{3}} \frac{1}{q^{2} k^{2}} \int_{-\pi}^{\pi} d \varphi f(p) f(k)\left(q k \cos \varphi-k^{2}\right)\left(q^{2}-q k \cos \varphi\right) \\
& =\frac{3 a_{c}^{2}}{16(2 \pi)^{2}} \int_{0}^{k_{d}} d q \int_{0}^{k_{d}} d k f(q) f(k) q k .
\end{aligned}
$$

All these integrals diverge at small momenta. This is due to the fact that we have taken the penetration depth $\lambda \equiv \lambda_{\perp}$ to be infinite. Upon reintroducing the lower cutoff $\lambda^{-1}$ the leading contribution (which diverges in the limit $\lambda \rightarrow \infty$ ) is found to be

$$
\begin{aligned}
& I_{1}=\frac{f^{2}}{2} \ln \left(k_{d} \lambda\right)+\mathcal{O}(1) \\
& I_{2}=-f \ln \left(k_{d} \lambda\right)+\mathcal{O}(1) \\
& I_{3}=\mathcal{O}\left(1,(\ln \lambda) / \lambda^{2}\right) .
\end{aligned}
$$

Hence one finds

$$
E_{\mathrm{VR}}^{(2)}=\frac{\phi_{0}^{2}}{8 \pi^{2} \lambda^{2}}\left[\frac{f^{2}}{2} \ln \left(k_{d} \lambda\right)-f \ln \left(k_{d} \lambda\right)+\frac{1}{2} f-\frac{3 f^{2}}{16}\right] .
$$

Since we are interested in the limit $\lambda \rightarrow \infty$ we must choose $f=1$ in order to cancel the $\ln \left(k_{d} \lambda\right)$ divergences in the final expression for the vacancy-free energy.

Now we have to look at the subleading terms. We set $f=1$ and do variational calculation for $c$ and $d$. One gets

$$
\begin{gathered}
c=\frac{4}{7 k_{d}} \approx 0.161 \sqrt{a_{c}}, \\
d=-\frac{10}{7 k_{d}^{2}} \approx-0.114 a_{c} .
\end{gathered}
$$

The result for the vacancy relaxation energy at constant lattice spacing becomes

$$
E_{\mathrm{VR}}^{(2)}=\frac{\phi_{0}^{2}}{32 \pi^{2} \lambda^{2}}\left[\ln \left(\frac{1}{k_{d}^{2} \lambda^{2}}\right)+\frac{265}{252}\right],
$$

which corresponds to a free energy of

$$
G_{D}=\left(\frac{\phi_{0}}{8 \pi \lambda}\right)^{2} 0.789
$$




\section{REFERENCES}

1. See, e.g., the reviews in Phenomenology and Applications of High-Temperature Superconductors, edited by K. Bedell et al. (Addison-Wesley, New York, 1991).

2. E. Brezin, D.R. Nelson, and A. Thiaville, Phys. Rev. B31, 7124 (1985).

3. M. Charalambous, J. Chaussy and P. Lejay, Phys. Rev. B45, 509 (1992).

4. H. Safar, P.L. Gammel, D.A. Huse, D.J. Bishop, J.P. Rice, and D.M. Ginsberg, Phys. Rev. Lett. 69, 824 (1992).

5. K.W. Kwok, S. Fleshler, U. Welp, V.M. Vinokur, J. Downey, G.W. Crabtree and M.M. Miller, Phys. Rev. Lett. 69, 3370 (1992).

6. A.I. Larkin and Y.M. Ovchinnikov, J. Low Temp. Phys. 34, 409 (1979).

7. D.R. Nelson and H.S. Seung, Phys. Rev. B39, 9153 (1989).

8. M.C. Marchetti and D.R. Nelson, Phys. Rev. B42, 9938 (1990).

9. F.R.N. Nabarro and A.T. Quintanilha, in Dislocations in Solids, edited by F.R.N. Nabarro (North-Holland, Amsterdam, 1980), Vol. 5.

10. N.W. Ashcroft and N.D. Mermin, Solid State Physics (Saunders College, Philadelphia 1976), Chapter 30 .

11. The dislocation loop in Fig. 1a differs from the vacancy-interstitial pair in Fig. 1b in that it relaxes shear stresses rather than relaxing a perpendicular current directly.

12. D.S. Fisher, M.P.A. Fisher, and D.A. Huse, Phys. Rev. B43, 130 (1991).

13. M. Feigel'man, V.B. Geshkenbein, and A.I. Larkin, Physica C167, 177 (1990).

14. V.M. Vinokur, P.H. Kes, and A.E. Koshelev, Physica C168, 29 (1990).

15. J.H. Hetherton, Phys. Rev. 176, 231 (1968).

16. A.F. Andreev and I.M. Lifshitz, Sov. Phys. JETP 29, 1107 (1969).

17. G. Chester, Phys. Rev. A2, 256 (1970). 
18. A.J. Leggett, Phys. Rev. Lett. 25, 1543 (1970).

19. I.E. Dzyaloshinskii, P.S. Kondratenko, and V.S. Levchenkov, Sov. Phys. JETP 35, 823, 1213 (1972).

20. K. Liu and M.E. Fisher, J. Low Temp. Phys. 10, 655 (1973).

21. M.P.A. Fisher and D.H. Lee, Phys. Rev. B39, 2756 (1989).

22. D.R. Nelson, in Ref. 1.

23. A.F. Andreev, "Quantum Crystals," in Progress in Low-Temperature Physics, Vol. VIII, edited by D.G. Brewer (North Holland, Amsterdam, 1982).

24. L.I. Glazman and A.E. Koshelev, Phys. Rev. B43, 2835 (1991).

25. (a) D.S. Fisher, B.I. Halperin and R. Morf, Phys. Rev. B20, 4692 (1979); This work is revised and extended in (b) E. Cockayne and V. Elser, Phys. Rev. B43, 623 (1991).

26. Several interstitials are in fact evident in the triangulation of decorated vortices in BSCCO of Fig. 2 in P. Gammel, D.J. Bishop and C.M. Murray, Phys. Rev. Lett. 64, 2312 (1990). These interstitials, however, may be an artifact of overdecoration (C.M. Murray, private communication). See also C.A. Bolle et al., Phys. Rev. Lett. 66, 112 (1991) for chains of interstitials which appear in magnetic fields tilted away from the $c$ axis. One possible explanation for the chains is a highly anisotropic interaction potential between interstitials.

27. A. Houghton, R.A. Pelcovits, and A. Sudbo, Phys. Rev. B40, 6763 (1989), and Refs. $13,14,25$, and 24.

28. D.S. Fisher in Ref. 1 and Ref. 25.

29. E.H. Brandt, Phys. Rev. B34, 6514 (1986); Phys. Rev. Lett. 63, 1106 (1989).

30. See Appendix B of D.R. Nelson and V. Vinokur, Phys. REv. B (in press).

31. E.H. Brandt, Phys. Rev. Lett. 69, 1105 (1992).

32. R.P. Feynman and A.R. Hibbs, Path Integrals and Quantum Mechanics, (McGrawHill, New York, 1965); R.P. Feynman, Statistical Mechanics (W.A. Benjamin, Reading, MA, 1972). 
33. See Ref. 32 and Appendix C of Ref. 30.

34. D.S. Fisher, Phys. Rev. B22, 1190 (1980).

35. D.R. Nelson, J. Stat. Phys. 57, 511 (1989).

36. A.L. Fetter, Phys. Rev. 147, 153 (1966); The values given for $A_{4}$ and $A_{6}$ by Fetter are corrected by the values given in the main text. We attribute the small difference to inaccuracies in the evaluation of the exponential integral function.

37. The actual stable vacancy configuration does not have this six-fold symmetry. The vacancy configuration with the six-fold symmetry is a saddle point. See also section 3.5 .

38. A.L. Fetter, P.C. Hohenberg and P. Pincus, Phys. Rev. 147, 140 (1966).

39. P.P. Ewald, Ann. Phys. (Leipzig) 54519 (1917); 54, 57 (1917); 64253 (1921).

40. M.P. Allen and D.J. Tildesley, Computer Simulations of Liquids, (Clarendon, Oxford, 1990).

41. We have used the polynomial and rational approximations 5.1.53 and 5.1.56 in M. Abramowitz, and I.A. Stegun, Handbook of Mathematical Functions, Dover Publ., Inc., New York (1965), page 231.

42. The error for the total energy of the bigger system is larger because we have used a square cell with a length to height ratio of $\frac{13}{15}$ instead of a hexagonal cell (see above). The error implied by this becomes larger for larger systems.

43. See, e.g., S. Coleman, Aspects of Symmetry (Cambridge University Press, 1987), Chapter 7; see also J.F. Currie et al., Phys. Rev. B22, 477 (1980).

44. The two-dimensional formula Eq. (2.14) gives $T_{m}=0.01 c_{0} d_{0}$, which is also less than $T_{d}$.

45. R.D. Kamien and D.R. Nelson, J. Stat. Phys. 71, 23 (1993); see Appendix B.

46. For a related treatment of hairpins in polymer nematics, see R.D. Kamien, P. Le Doussal and D.R. Nelson, Phys. Rev. A45, 8727 (1992). 
47. Z. Tesanovic, Johns Hopkins University preprint.

48. Similar issues are discussed in E.H. Brandt, J. Low. Temp. Phys. 42, 557 (1981); ibid 44, 33 (1981); ibid 44, 59 (1981).

49. D. Huse and Majumdar, AT\&T Bell Laboratories preprint; see also C.M. Marchetti and D.R. Nelson, Physica C174, 40 (1991).

50. D.R. Nelson and P. Le Doussal, Phys. Rev. B42, 10112 (1990).

51. E.M. Chudnovsky, Phys. Rev. B40, 11355 (1989).

52. M.V. Feigel'man, V.B. Geshkenbein, A.I. Larkin and V.M. Vinokur, Phys. Rev. Lett. 63, $2302(1989)$. 


\section{TABLE 1}

Estimates for Melting and Defect Unbinding Transitions

\begin{tabular}{ccc}
\hline \hline Regime & $T_{m}(B)$ & $T_{d}(B)$ \\
\hline$B_{\times} \lesssim B$ & $0.5 \varepsilon_{0} d_{0} / 8 \pi \sqrt{3}$ & const. $\times \varepsilon_{0} d_{0} / \ln (2 \pi B / B \times$ \\
$B_{c 1} \ll B \lesssim B_{\times}$ & $c_{L}^{2} \sqrt{\varepsilon_{0} \tilde{\varepsilon}_{1}}\left(\phi_{0} / B\right)^{1 / 2}$ & $c_{3} \sqrt{\varepsilon_{0} \tilde{\varepsilon}_{1}}\left(\phi_{0} / B\right)^{1 / 2}$ \\
$B \lesssim B_{c 1}$ & $c_{L}^{2} \sqrt{\varepsilon_{0} \tilde{\varepsilon}_{1}} \lambda_{\perp}\left(\frac{B_{c 1}}{B}\right) e^{-\frac{1}{2}\left(B_{c 1} / B\right)^{1 / 2}}$ & const. $\times \sqrt{\varepsilon_{0} \tilde{\varepsilon}_{1}} \lambda_{\perp}\left(\frac{B_{c 1}}{B}\right) e^{-\frac{1}{2}\left(B_{c 1} / B\right)^{1 / 2}}$ \\
\hline \hline
\end{tabular}


TABLE 2

Exact and numerically calculated energy per particle of the perfect flux line lattice for different system sizes. $N$ is the number of flux lines. The energies are measured in units of $E_{0}=2 \varepsilon_{0}$.

\begin{tabular}{ccc}
\hline \hline$N$ & $E_{\text {exact }}$ & $E_{\text {num }}$ \\
\hline 30 & 0.85583194 & 0.85583216 \\
120 & 1.20240554 & 1.20240652 \\
270 & 1.40513809 & 1.40514051 \\
480 & 1.54897913 & 1.54898290 \\
750 & 1.66055090 & 1.66055687 \\
1080 & 1.75171168 & 1.75172015 \\
1470 & 1.82878705 & 1.82879859 \\
\hline \hline
\end{tabular}




\section{TABLE 3}

Defect energies at constant line density for the symmetric vacancy (V6), "squeezed" vacancy (V2), edge interstitial (EI), centered interstitial (CI), split edge interstitial (SEI), and split centered interstitial (SCI) configurations. The results are obtained from a molecular dynamics type of calculation for different system sizes with $\mathrm{N}$ flux lines. The energies are measured in units of $E_{0}=2 \varepsilon_{0}$.

\begin{tabular}{ccccccc}
\hline \hline$N$ & $E_{\mathrm{V} 6}$ & $E_{\mathrm{V} 2}$ & $E_{\mathrm{EI}}$ & $E_{\mathrm{CI}}$ & $E_{\mathrm{SEI}}$ & $E_{\mathrm{SCI}}$ \\
\hline 30 & 0.11857 & 0.11394 & 0.07156 & 0.07218 & 0.07156 & 0.07166 \\
120 & 0.12204 & 0.10892 & 0.07358 & 0.07274 & 0.07359 & 0.07295 \\
270 & 0.12381 & 0.10825 & 0.07392 & 0.07293 & 0.07392 & 0.07310 \\
480 & 0.12416 & 0.10783 & 0.07403 & 0.07299 & 0.07402 & 0.07315 \\
750 & 0.12429 & 0.10781 & 0.07408 & 0.07304 & 0.07405 & 0.07319 \\
1080 & 0.12434 & 0.10772 & 0.07410 & 0.07309 & 0.07411 & 0.07324 \\
1470 & 0.12437 & 0.10780 & 0.07412 & 0.07307 & 0.07411 & 0.07325 \\
\hline \hline
\end{tabular}




\section{Figure Captions:}

Fig.1a: Dislocation loop in a flux line solid. Dashed lines represent vortices just behind the plane of the figure. Such loops lie in the plane spanned by their Burgers vector and the $z$-axis. The orientation of the three triangles is the same, showing that the loop has only a small effect on the orientational order.

Fig.1b: Vacancy-interstitial pair in a flux line solid. Dashed lines represent vortices just behind the plane of the figure. Unlike the dislocation loop in Fig.1a, this loop is not constrained to lie in a single plane.

Fig.2: Vacancy line (thick dashed curve) meandering through a vortex crystal. The full lines show the flux lines which are in the same plane as the meandering vacancy. The dashed lines represent the flux lines in the neighboring plane.

Fig.3: Lowest energy contribution to the order parameter correlation function on the solid phase (1.4), this inserts a flux head and tail into the crystalline vortex array. Dashed lines represent a row of vortices slightly behind the plane of the page. A vacancy is created at "time" $z$, propagates and is destroyed at "time" $z^{\prime}$. The energy of the "string" defect connecting the head to the tail increases linearly with $\left|\vec{r}_{\perp}-\vec{r}_{\perp}^{\prime}\right|$ and leads to the exponential decay of $G\left(\vec{r}_{\perp}, \vec{r}_{\perp}^{\prime}, z, z^{\prime}\right)$. Physically, this represents confinement of the magnetic monopoles represented by the flux head and tail.

Fig.4: Two distinct scenarios for vortex crystal melting with increasing temperature. In type I melting a first order transition separates a line crystal with $\rho_{\vec{G}} \neq 0$ from a flux liquid with $\psi_{0} \neq 0$. In type II melting, both order parameters are nonzero in the intermediate "supersolid" phase.

Fig.5: Schematic phase diagram of a clean high temperature superconductor. The "supersolid" phase is shown as the shaded region. In the presence of random pinning, a possible 
vortex glass transition, at which the resistance vanishes, would roughly follow the melting boundary for low fields and the crystal-supersolid boundary in high fields.

Fig.6: Initial defect configurations for a symmetric vacancy, centered interstitial, edge interstitial, split centered and split edge interstitial used in the numerical calculation of the defect formation energies.

Fig.7: Starting from the initial symmetric vacancy configuration, shown as squares, the flux line lattice relaxes first into a symmetric configuration, shown by the diamonds. This configuration, however, is just a saddle point and unstable against squeezing the vacancy along one of the three symmetry axis. One of three degenerate stable final configurations is represented by the circles.

Fig.8: Relaxed configurations for centered (squares) and edge (circle) interstitial. The edge interstitial is unstable with respect to a "buckling" mode perpendicular to the edge of the triangle. The final stable configuration is the centered interstitial configuration.

Fig.9a: System size dependence of the formation energies for centered (solid line), split centred (dot-dashed line) and edge (dashed line) interstitials. The energy in units of $E_{0}$ is plotted versus the total number of flux lines $N$. The lowest energy configuration is the centered interstitial.

Fig.9b: System size dependence of the formation energies for symmetric (solid line), and "crushed" (solid line) vacancy. The energy in units of $E_{0}$ is plotted versus the total number of flux lines $N$. The symmetric vacancy configuration (6-fold symmetry) is unstable against squeezing it along one of the three symmetry axis. The final stable configuration is the "crushed" vacancy with only a two-fold symmetry.

Fig.10: Relaxed configuration for an edge (open circles) and split edge (open squares) interstitial. The two cofigurations differ by a shift along the edge of the triangle. Because of their energy difference being small gliding along the edge of an triangle is a low energy 
process. Note, however, that both configurations are unstable to buckling, resulting in the centered interstitial configuration.

Fig.11a: Interaction energy of two centered interstitials in units of $E_{0}$ versus the distance measured in units of the lattice constant $a_{0}$ for two different directions. (i) The vector connecting the two centered interstitials points along $\vec{a}$, one of the basis vectors of the unit cell (solid line). (ii) The vector connecting the two centered interstitials points along $\vec{b}$, which is perpendicular to $\vec{a}$ (dashed line). Whereas the interaction in the a-direction is attractive at small distances it is repulsive in the perpendicular b-direction. In the b-direction there is a minimum in the interaction energy at a distance of $d_{\min } \approx 2.5 a$.

Fig.11b: Interaction energy of two "crushed" vacancies in units of $E_{0}$ versus the distance measured in units of the lattice constant $a$. The vector connecting the vacancies is pointing a) along the two-fold symmetry axis of the vacancy (solid line) and b) perpendicular to it (dashed line).

Fig.12: Basis vectors $\left\{\vec{\delta}_{i}\right\}$ for the honeycomb lattice of centered interstitial sites with lattice constant $b$. The $A$ and $B$ sublattices are indicated by open and closed circles. 